\title{
Inefficient exploitation of accessory receptors reduces the sensitivity of chimeric antigen receptors
}

\author{
Jake Burton $^{1}$, Jesús A. Siller-Farfán ${ }^{1 \uparrow}$, Johannes Pettmann ${ }^{1}$, Benjamin Salzer ${ }^{1 \dagger}$, \\ Mikhail Kutuzov ${ }^{1}$, P. Anton van der Merwe ${ }^{1}$, Omer Dushek ${ }^{1}{ }^{1}$ \\ ${ }^{1}$ Sir William Dunn School of Pathology, University of Oxford, OX1 3RE, Oxford, UK \\ †Present address: St. Anna Children's Cancer Research Institute (CCRI), 1090, Vienna, Austria \\ ${ }^{\top}$ Corresponding authors
}

\begin{abstract}
Chimeric antigen receptors (CARs) can re-direct $\mathrm{T}$ cells to target abnormal cells but their activity is limited by a profound defect in antigen sensitivity, the source of which remains unclear. Here, we show that CARs have a $>100$-fold lower antigen sensitivity compared to the TCR when antigen is presented on antigen-presenting-cells, but nearly identical sensitivity when antigen is presented as purified protein in isolation. Given that the TCR uses other, accessory, receptors to achieve high sensitivity, we screened prominent accessory receptors by presenting their purified ligands together with antigen. We found that ligating the adhesion receptor CD2 or LFA-1 improved antigen sensitivity for the TCR by $>100$-fold, whereas for CARs the improvement was $<10$-fold. We reproduced these findings using target cells where the CD2 and/or LFA-1 interaction were abrogated. Sensitivity can be partially restored by fusing the CAR variable domains to the TCR CD3 $\varepsilon$ subunit (also known as a TRuC) and fully restored when exchanging the TCR $\alpha \beta$ variable domains for those of the CAR (also known as a STAR). Our study localises the defect in CAR sensitivity to inefficient use of accessory receptors and suggests approaches to increase sensitivity.
\end{abstract}




\section{Introduction}

Adoptive cell transfer (ACT) of genetically engineered $\mathrm{T}$ cells expressing Chimeric Antigen Receptors (CARs) is a clinically approved cancer therapy for haematological malignancies $(1,2)$. CARs are synthetic receptors that are typically generated by the fusion of an antibody-derived, antigen-binding single-chain variable fragment $(\mathrm{scFv})$ with intracellular signalling motifs from the cytoplasmic tails of the T cell receptor (TCR) complex. Although CAR-T cells targeting the surface antigens CD19, CD20, and B cell maturation antigen (BCMA or CD269) on malignant B cells show unprecedented clinical benefits, a large fraction of patients receiving these therapies relapse with B cells expressing low or undetectable levels of these surface antigens (3-8). It is now appreciated that CARs require 100 to 1000 -fold higher antigen densities to induce $\mathrm{T}$ cell activation compared to the native TCR and this reduced antigen sensitivity has been observed for distal T cell responses (9) and for CAR proximal signalling $(10,11)$. The mechanism underlying the defect in CAR antigen sensitivity remains unclear.

There has been work focused on improving various aspects of the original CAR architecture, including changes to the stalk/hinge and the cytoplasmic signalling domains. There are several commonly used hinges, including the CD8a, CD28, and IgG1 and currently, the majority of CARs use the $\zeta$-chain from the TCR complex on its own for signalling ( $1^{\text {st }}$ generation) or in combination with the CD28 (or 4-1BB) signalling domain ( $2^{\text {nd }}$ generation $)(12-15)$. Recently, it has been suggested that replacing the $\zeta$-chain with the $\varepsilon$-chain of the TCR complex would improve CAR function $(11,16,17)$. Moreover, it has been suggested that TCRlike chimeric receptors improve on the basic CAR design; by fusing the scFv directly to the extracellular domain of $\mathrm{CD} 3 \varepsilon$ (termed TruC) (18) or by replacing the variable domains of the TCR with those in the scFv (termed STAR) (19). However, accurate measurements of the relative antigen sensitivities of CARs have yet to be performed partly because it is difficult to vary the surface density of antigens on target cells.

Improving the antigen sensitivity of CARs can be inspired by the the native TCR. The TCR is known to have remarkable antigen sensitivity; it is able to recognise even a single peptide antigen on majorhistocompatibility-complex (pMHC) (20). It achieves this high sensitivity using diverse mechanisms (21). These mechanisms include having multiple immunoreceptor tyrosine-based activation motifs (ITAMs) (22, 23), using the TCR co-receptors CD4 or CD8 (24, 25), and exploiting TCR accessory receptors such as LFA-1 (26) and CD2 (27). Although CD2 has been shown to affect T cell activation by $1^{\text {st }}$ generation CARs (28), its effect on antigen sensitivity has not been determined.

Here, we directly compared the antigen sensitivity of the TCR with commonly used CAR architectures. We found that CARs exhibit a $>100$-fold lower antigen sensitivity when antigen is presented on cells but nearly identical sensitivities when antigen is presented as purified protein. We show that adding purified ligands to the accessory receptors CD2 and LFA-1 dramatically enhances the sensitivity of the TCR to antigen but only modestly increases the sensitivity of CARs: this finding was reproduced using cells presenting antigen with or without the CD2 and LFA-1 ligands. We show that the antigen sensitivity of CARs can be partially restored using a TruC (which engages the full complement of TCR signalling subunits) and fully restored using a STAR, which closely reproduce the structural features of the TCR. Our work provides insights into the mechanisms involved in antigen recognition by CARs and TCRs, and suggests ways to improve CAR sensitivity. 


\section{Results}

\section{Standard CAR designs exhibit reduced sensitivity compared to the TCR when antigen is presented on APCs but not when presented in isolation}

To compare the antigen sensitivity of TCRs and CARs, we utilised the C9V variant (9V) of the NY-ESO$1_{157-165}$ cancer testis peptide antigen expressed on HLA-A*02:01 that is recognised by the 1 G4 TCR $(29,30)$ and the D52N scFv (31) (Fig. 1A). We produced five commonly used CAR designs by fusing the D52N scFv to either the CD28, CD8a, or the IgG1 hinge coupled to either the TCR $\zeta$-chain alone ( $1^{\text {st }}$ generation) or in combination with the CD28 signalling chain ( $2^{\text {nd }}$ generation). Using a standard protocol similar to those employed in adoptive $\mathrm{T}$ cell therapy (32), we generated a panel of primary human $\mathrm{CD} 8^{+} \mathrm{T}$ cells expressing each of these six antigen receptors and presented them with the HLA-A*02:01+ T2 target cell line pulsed with different concentration of antigen (Fig. 1B). We found that T cells expressing the 1G4 TCR were able to kill target cells (as measured by LDH release) and upregulate the CD69 activation marker at $>100$-fold lower antigen concentrations compared to CARs (Fig. 1C,D). Comparing the CARs, we found that the addition of the CD28 signalling domain in $2^{\text {nd }}$ generation CARs did not impact antigen sensitivity, which is consistent with a previous report (33), and that the CD28 and CD8a hinges produced a modest enhancement in sensitivity compared to the IgG1 hinge. The reduced sensitivity could not readily be explained by receptor expression because the CARs were expressed at the same level (or higher) compared to the TCR (Figure S1). This $>100$-fold higher sensitivity of the TCR is consistent with two previous reports $(9,10)$ that utilised different hinges and different signalling chains (2nd generation CARs with 4-1BB coupled to the $\zeta$-chain), validating our antigen receptor system and suggesting that reduced sensitivity is a general feature of CARs.

The high antigen sensitivity of the TCR (20) is known to be achieved by a number of different mechanisms, including engagement of accessory receptors with their ligands on APCs (21). To determine whether accessory receptor/ligand interactions contributed to the difference in antigen sensitivity, we stimulated TCR and CAR expressing T cells using plate-immobilised purified pMHC. This reductionist system has been previously used to display defined ligands to T cells (30, 34-37). In striking contrast to the $>100$-fold difference in sensitivity when antigen was presented on APCs, the TCR and CARs displayed similar antigen sensitivities when recognising purified antigen, with the largest difference observed for the $1^{\text {st }}$ generation CD8a CAR (D52N-CD8a-z, which was only 3.5-fold less sensitive than the TCR). 


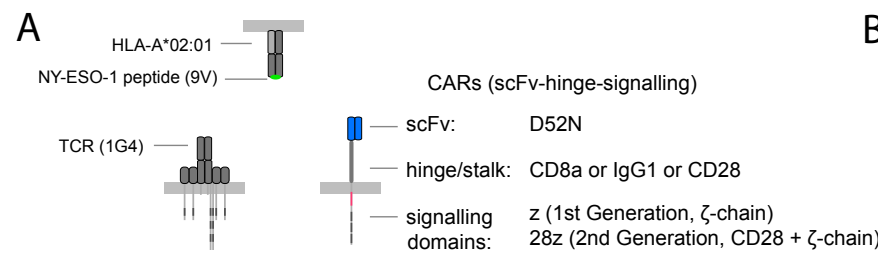

B

C

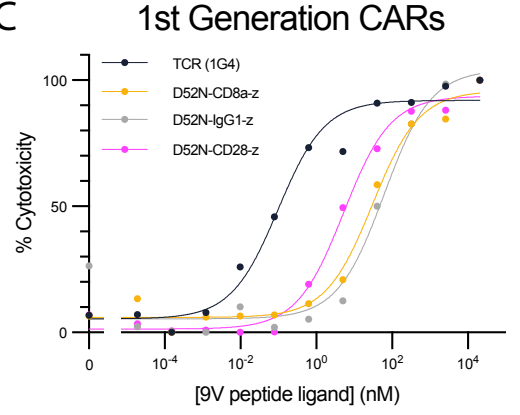

D
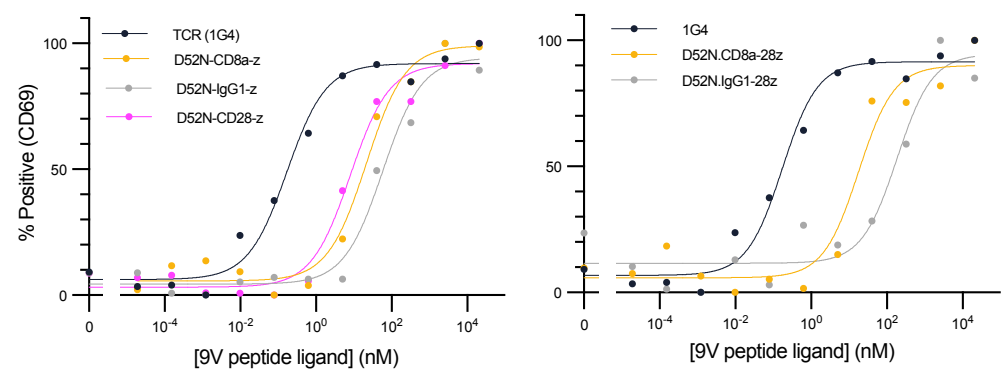
CAR (or TCR) transduced
$\mathrm{CD} 8^{+} \mathrm{T}$ cells

Solid-phase stimulation

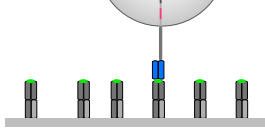

Recombinant biotinylated pMHC

presented on streptavidin-coated plates
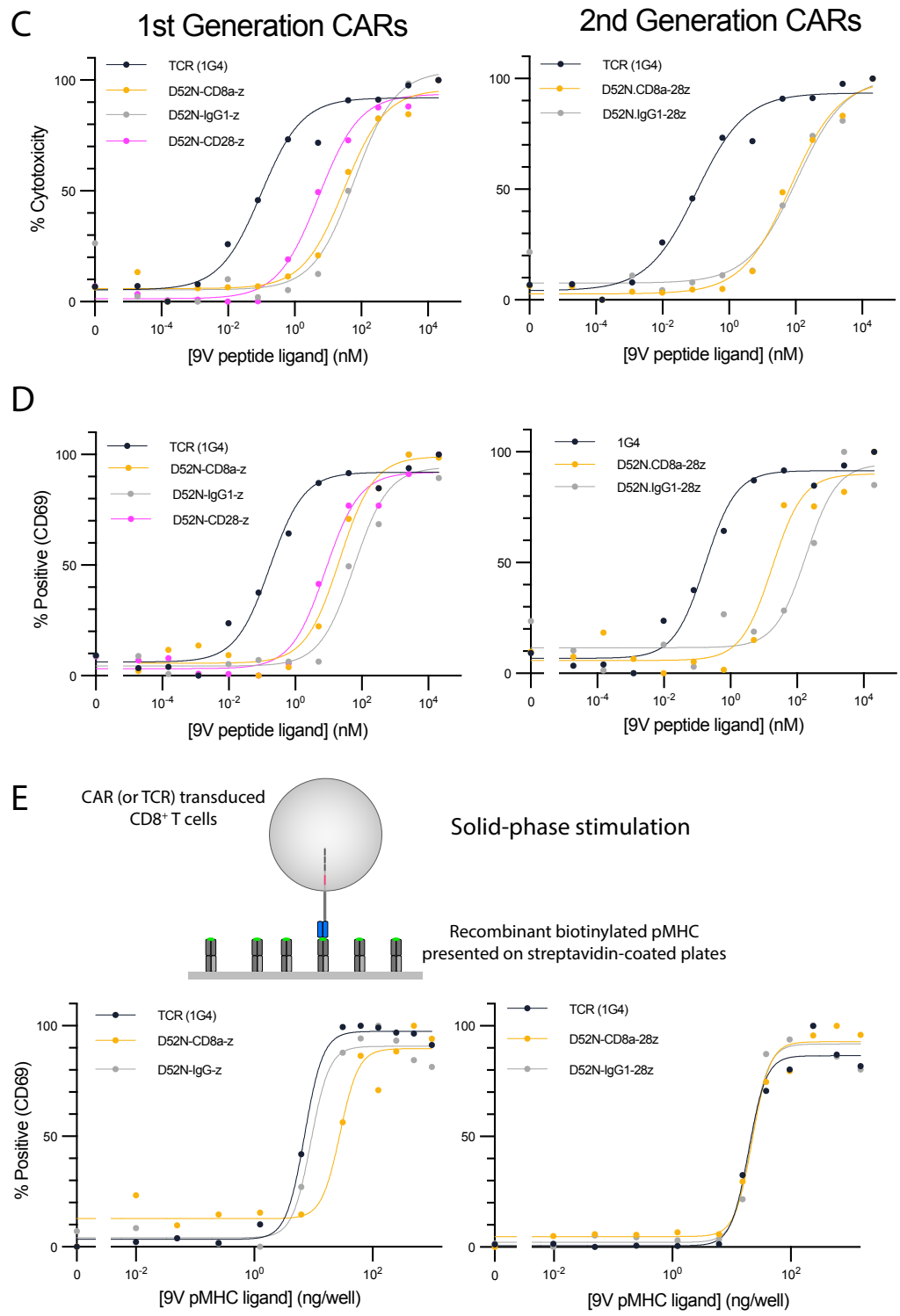

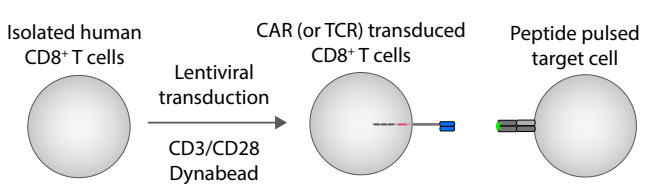

Expansion Co-culture (24 hour)
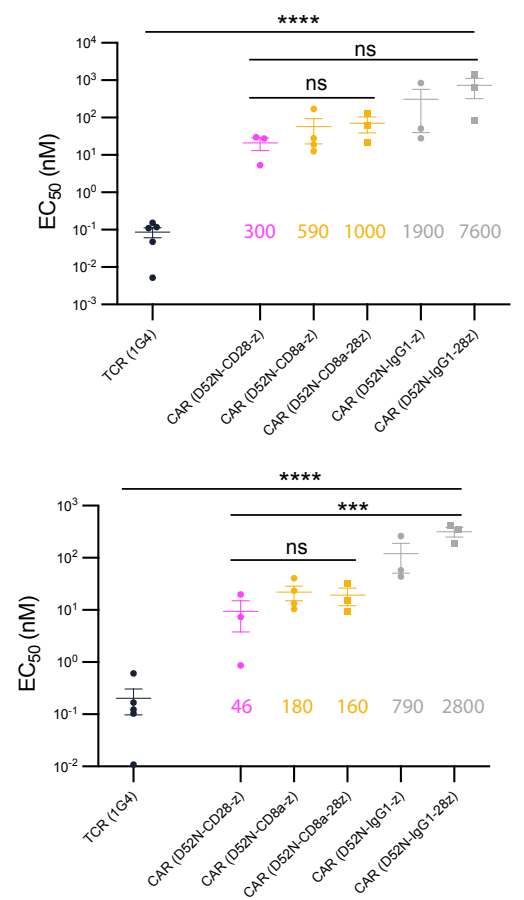

Figure 1: CARs show reduced sensitivity compared to the TCR when antigen is presented on APCs but not when presented as purified protein. (A) Schematic of antigen receptors. The $1 \mathrm{G} 4 \mathrm{TCR}$ and the D52N scFv both recognise the 9V NY-ESO-1 peptide antigen presented on HLA-A*02:01. CARs using the CD8a hinge contain the CD8a transmembrane domain whereas CARs using the IgG1 or CD28 hinges contain the CD28 transmembrane domain. (B) Schematic of APC stimulation system. (C-D) Representative dose-response showing (C) LDH release and (D) surface expression of CD69 for the TCR and the indicated $1^{\text {st }}$ and $2^{\text {nd }}$ generation CARs along with $\mathrm{EC}_{50}$ values from independent samples. (E) Representative doseresponse when purified biotinylated $9 \mathrm{~V}$ pMHC is presented on streptavidin-coated plates. The $\mathrm{EC}_{50}$ values are compared using (C,D) one-way ANOVA or (E) one-sample t-test for a hypothetical mean of $1.0 \mathrm{on} \log$ transformed values. Abbreviations: $*=\mathrm{p}$-value $\leq 0.05, * *=\mathrm{p}$-value $\leq 0.01, * * *=\mathrm{p}$-value $\leq 0.001, * * * *=$ $\mathrm{p}$-value $\leq 0.0001$. 


\section{Ligands to the adhesion receptors CD2 and LFA-1 increase the antigen sensitivity difference between the TCR and CARs}

Our finding that the $>100$-fold higher sensitivity of TCR compared to CARs is eliminated in a reductionist system provided an opportunity to explore the underlying mechanism (Fig. 1). A key difference between APCs and our reduced system is the presence of other, accessory, receptor/ligand interactions, such as the prominent $\mathrm{CD}^{+} \mathrm{T}$ cell co-stimulation receptors CD2, LFA-1, CD28, CD27, and 4-1BB (Fig. 2). To determine whether engagement of these receptors can account for the sensitivity differences, we screened for their ability to increase antigen sensitivity by including purified forms of their ligands alongside pMHC. We used a concentration of $250 \mathrm{ng} /$ well of each ligand, which we previously showed had dramatic impacts on $\mathrm{T}$ cell responses $(30,36,38)$.

In the case of the TCR, we found that addition of CD58 (ligand for CD2) or ICAM-1 (ligand for LFA1) led to a $~ 100$-fold increase in antigen sensitivity, whereas addition of CD86 (ligand for CD28), CD70 (ligand for CD27), or 4-1BBL (ligand for 4-1BB) led to modest $<5$-fold changes in sensitivity (Fig. 2B, left). This is consistent with our previous studies on the effects of engagement of CD2 and LFA-1 (39), 41BB and CD27 (38), and CD28 (36). In contrast, we found that addition of these ligands had only a modest impact on the sensitivity either $1^{\text {st }}$ or $2^{\text {nd }}$ generation CARs (Fig. 2B-D). The largest increase was observed for the $1^{\text {st }}$ generation CD8a hinge CAR (D52N-CD8a-z) with the addition of ICAM-1 producing a 7-fold increase in sensitivity (Fig. 2D). Therefore, the TCR and CARs have similar antigen sensitivities to purified pMHC but differences in antigen sensitivities when ligands to accessory receptors are expressed with the largest impact observed when including ligands to CD2 or LFA-1 (Fig. 2E).

Previously, it has been reported that some CARs can induce tonic signals that lead to $\mathrm{T}$ cell dysfunction/exhaustion by various mechanisms, including changing the expression of surface receptors and/or unique signalling or transcriptional states (40-42). This raised the possibility that tonic signals may be limiting antigen sensitivity. We first confirmed that the expression level of each co-stimulation receptor was identical between TCR and CAR-transduced T cells (Fig. S2A). Second, the expression of the co-inhibition receptors and exhaustion markers LAG-3 and PD-1 were similar between TCR and CAR-transduced T cells with $<2$-fold increase in TIM-3 (Fig. S2B). Next, we showed that transduction of a CAR did not affect the sensitivity of an orthogonal TCR recognising a viral peptide, with or without the CD2 ligand (Fig. S2C). Taken together, this suggests a CAR-proximal defect in antigen sensitivity rather then a dysfunctional $\mathrm{T}$ cell state.

Supra-physiological affinities can impair TCR signalling and reduce antigen sensitivity $(35,43,44)$ and lowering the affinity of CARs has been shown to improve their in vivo activity (45). The D52N scFv binds the $9 \mathrm{~V}$ pMHC with a $\sim 50$-fold higher affinity than the $1 \mathrm{G} 4 \mathrm{TCR}$ at $37^{\circ} \mathrm{C}$ (Fig. S3A) raising the possibility that higher-affinity may be impairing CAR signalling. To address this, we identified a lower-affinity antigen that bound the D52N scFv with the same affinity as the TCR binding the 9V pMHC (Fig. S3A; 4A pMHC). Using this antigen we showed that the difference in antigen sensitivity between the TCR and CAR increased rather than decreased when using matched affinity pMHCs (Fig. S3B-E). Lastly, we confirmed that the $\mathrm{CD} 8$ co-receptor is not interfering with CAR antigen recognition and is not contributing to the difference in antigen sensitivities (Fig. S4).

In summary, the antigen sensitivities of the TCR and CARs are similar when they recognise purified antigen in isolation. However, in the presence of ligands to the adhesion receptors CD2 or LFA-1, the antigen sensitivity of the TCR is dramatically enhanced compared to CARs. This difference in antigen sensitivity is 
bioRxiv preprint doi: https://doi.org/10.1101/2021.10.26.465853; this version posted October 28, 2021. The copyright holder for this preprint (which was not certified by peer review) is the author/funder, who has granted bioRxiv a license to display the preprint in perpetuity. It is made available under aCC-BY 4.0 International license.

not a result of differences in accessory receptor expression, tonic-signalling induced exhaustion, affinity, or the role of the CD8 co-receptor. 
A

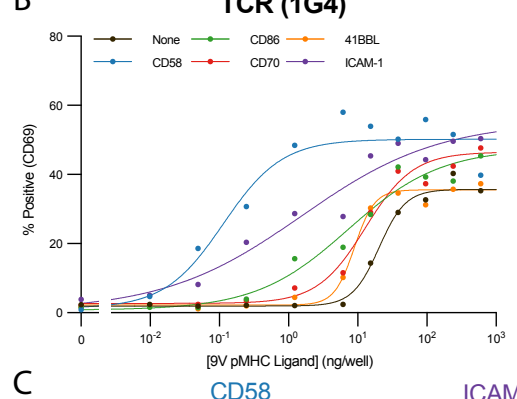

B

C

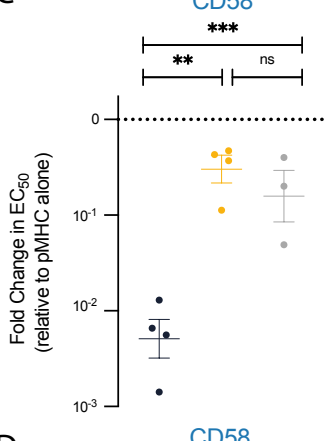

D $\quad$ CD58

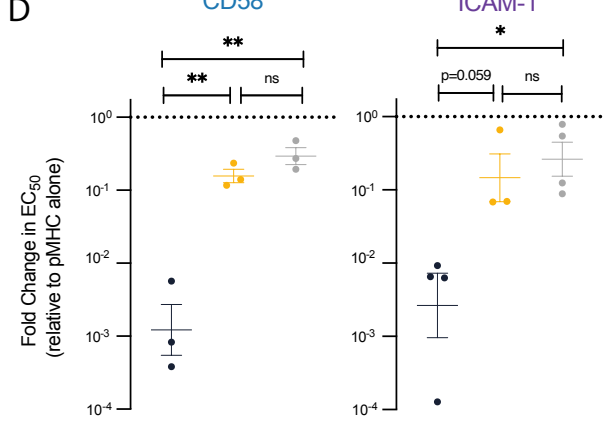

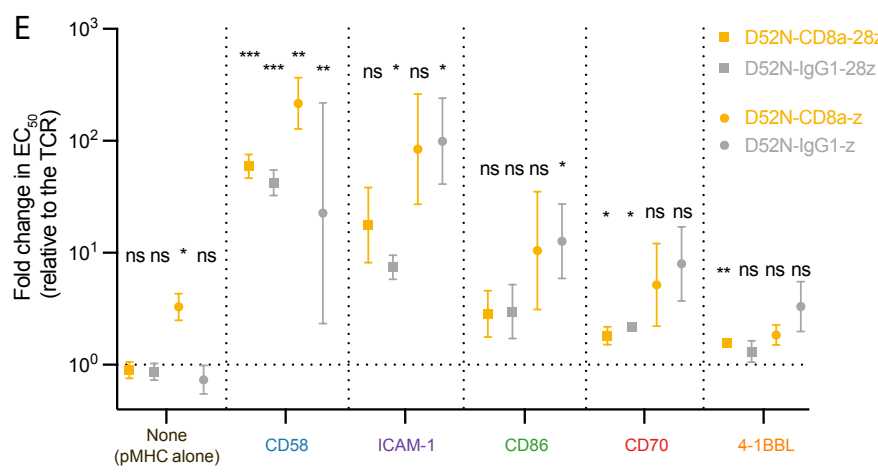

D52N-CD8-28z D52N-lgG1-28z

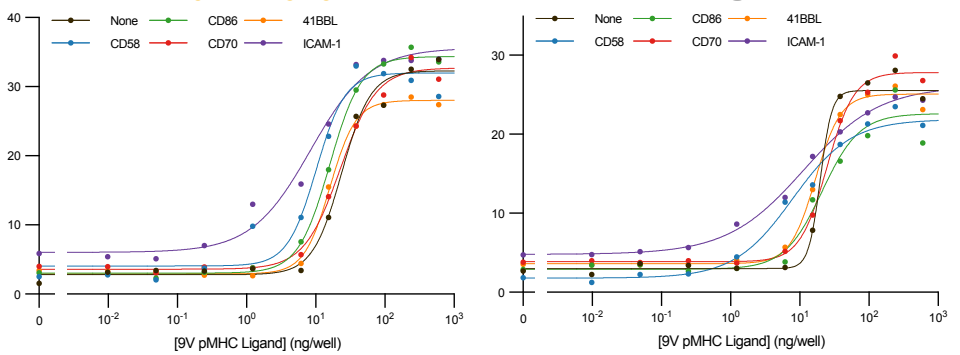

CD86

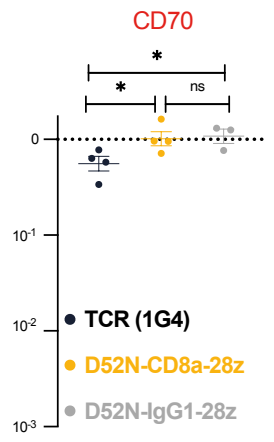

pMHC Ligand] (ng/well)
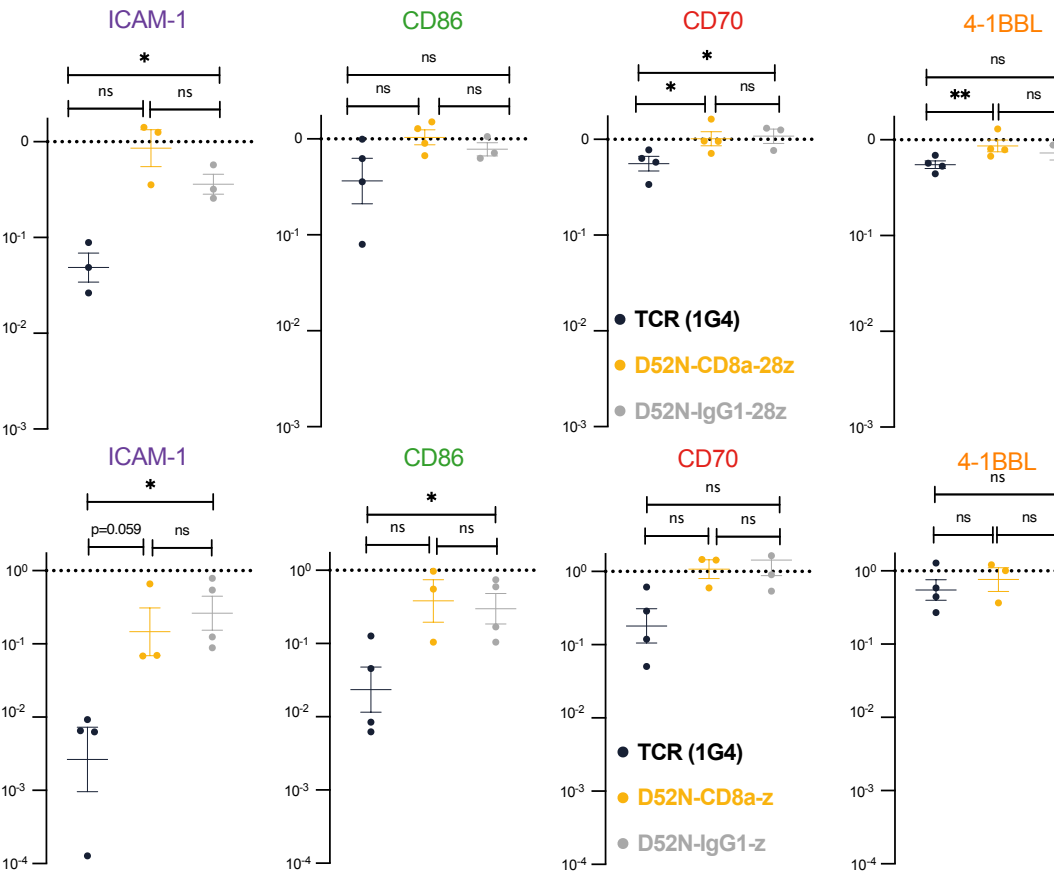
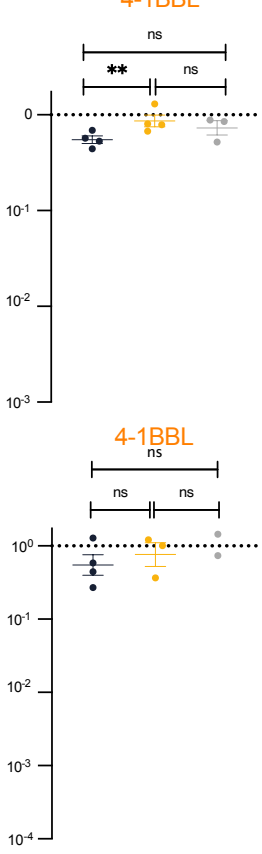

Figure 2: Systematic co-presentation of purified ligands to co-stimulation receptors identifies that CARs fail to exploit the adhesion receptors CD2 and LFA-1 to increase antigen sensitivity. (A) Schematic of screened accessory receptors and their ligands. (B) Representative pMHC dose-response curves with a fixed concentration of $250 \mathrm{ng} /$ well of the indicated accessory receptor ligand for the TCR (left) and CARs (middle, right). (C-D) Fold-change in $\mathrm{EC}_{50}$ for the indicated ligand relative to antigen alone for (C) $2^{\text {nd }}$ and (D) $1^{\text {st }}$ generation CARs. (E) Mean fold-change in $\mathrm{EC}_{50}$ for the indicated CARs relative to the TCR mean, with antigen alone or the indicated accessory receptor ligand. The fold-change is compared using a one-sample t-test to a hypothetical value of 0 on $\log$-transformed data. The $\mathrm{EC}_{50}$ values are compared using a paired t-test on log-transformed data. Abbreviations: $*=p$-value $\leq 0.05, * *=$ p-value $\leq 0.01, * * *=\mathrm{p}$-value $\leq 0.001, * * * *=\mathrm{p}$-value $\leq 0.0001$. 


\section{Abrogating the CD2 and LFA-1 interaction reduces the antigen sensitivity difference between the TCR and CARs}

The antigen sensitivities of the TCR and CARs were similar when recognising purified antigen in isolation (Fig. 1) but exhibited large differences with the addition of purified ligands to CD2 or LFA-1 (Fig. 2). To investigate the role of these adhesion interactions in target cell recognition, we utilised the HLA-A*02:01+ U87 glioblastoma cell line, which expresses CD58 and ICAM-1 (Fig. S5A-B). We used blocking antibodies targeting CD58 or ICAM-1 (Fig. 3A-D) and CRISPR to produce knockout cell lines (Fig. S5A-B, Fig. 3E$\mathrm{H})$. In both cases, we found that abrogating these interactions modulated $\mathrm{T}$ cell activation as measured by CD69 and 4-1BB expression. As with the T2 cells, we found a $\sim 100$-fold difference in antigen sensitivity between the TCR and CAR using the isotype control or the parental U87 line but this difference decreased to $\sim 20$-fold when abrogating both the CD2 and LFA-1 interaction (Fig. 3C-D,G-H, left). The decrease in this difference was largely a result of the decrease in antigen sensitivity by the TCR when abrogating these interactions (Fig. 3C-D,G-H, right). We note that the antigen sensitivity of the TCR remained 20fold higher than the CAR when abrogating both CD2 and LFA-1 interactions. This suggests that other mechanisms, including potentially other receptor/ligand interactions, continue to contribute and indeed, the U87 cell line expresses other ligands to LFA-1 (Fig. S5C). In summary, experiments using artificial surfaces and cell-presented antigens show that the TCR can more efficiently exploit the adhesion receptors CD2 and LFA-1 resulting in higher antigen sensitivities compared to CARs. 
A
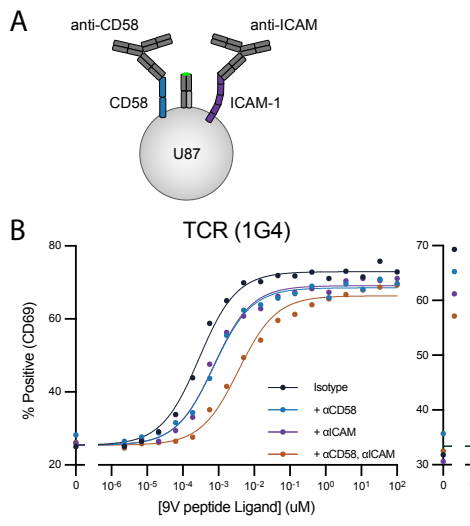

C CD69

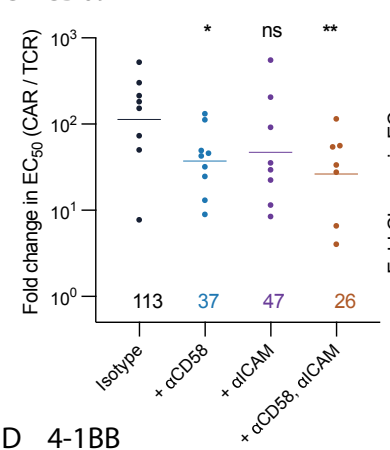

$$
\text { (10.0. }
$$
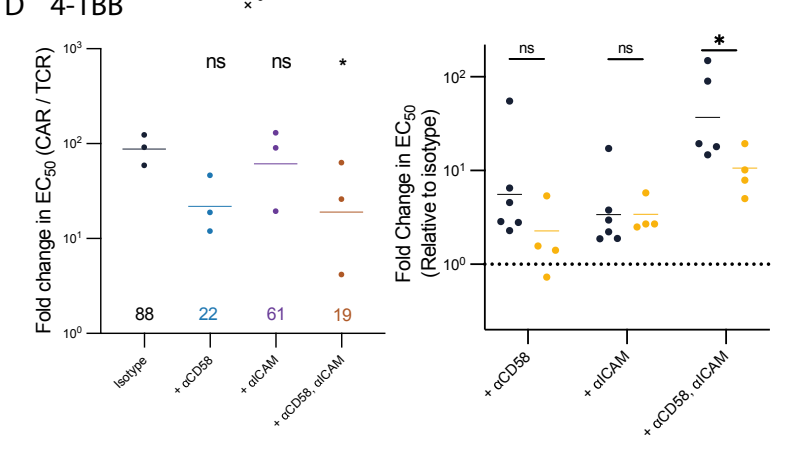

$\mathrm{E}$
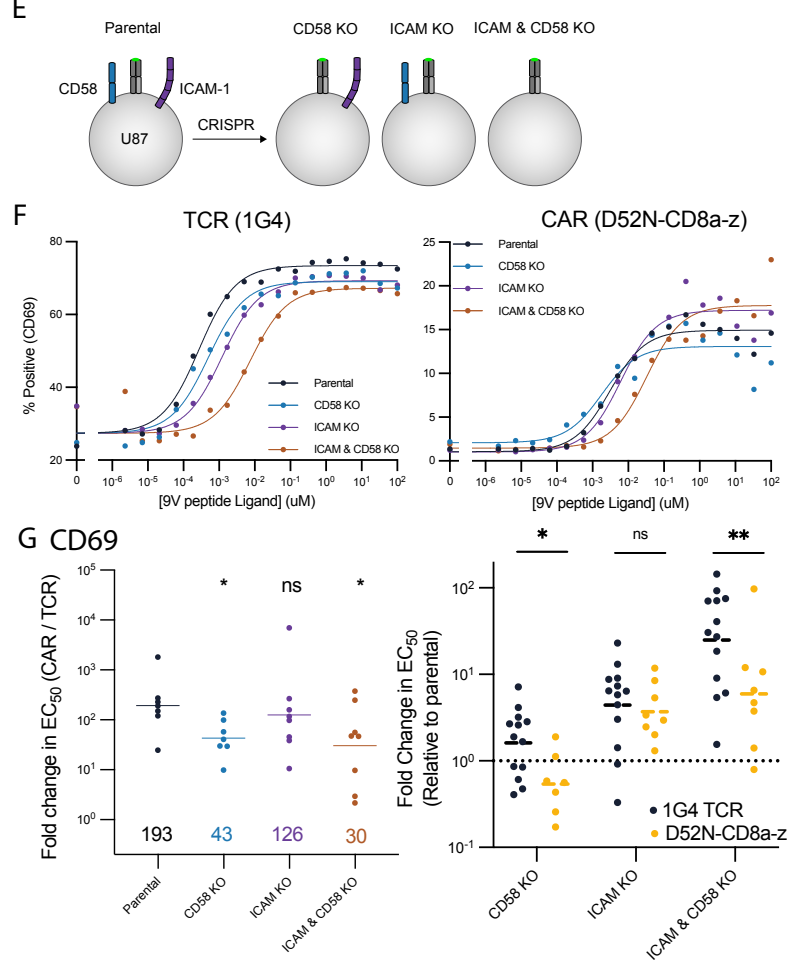

H $4-1 B B$

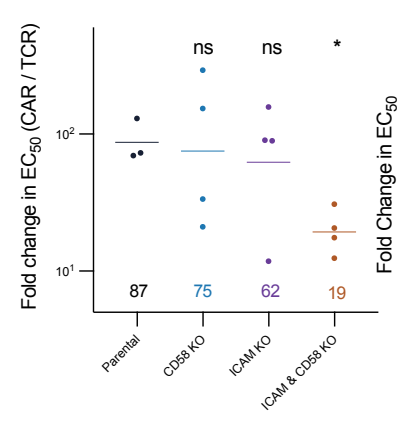

Figure 3: Abrogating the CD2 and LFA-1 adhesion interaction disproportionately impacts antigen sensitivity for the TCR compared to the CAR. (A) Schematic of CD58 and ICAM-1 blocking experiment on the HLA-A2+ glioblastoma U87 target cell line. (B) Representative dose-response curves for the indicated blocking conditions for the TCR (left) and CAR (right). (C-D) Fold-change in $\mathrm{EC}_{50}$ between the CAR and TCR (left) or relative to the isotype (right) for (C) CD69 and (D) 4-1BB. (E) Schematic of CD58 and ICAM-1 knockout experiments. (F) Representative dose-response curves for the indicated target cell lines for the TCR (left) and CAR (right). (G-H) Fold-change in $\mathrm{EC}_{50}$ between the CAR and TCR (left) or relative to the isotype (right) for (G) CD69 and (H) 4-1BB. The fold-change between the TCR and CAR is compared using a two-sample t-test to the isotype or parental line condition (left panel in $\mathrm{C}, \mathrm{D}, \mathrm{G}, \mathrm{H}$ ) or directly between the TCR and CAR (right panels in C, D, G, H) on log-transformed values. Fits are constrained by sharing the hill coefficient. Abbreviations: $*=$ p-value $\leq 0.05, * *=p$-value $\leq 0.01, * * *=p$-value $\leq 0.001$, $* * * *=\mathrm{p}$-value $\leq 0.0001$.

\section{STARs display TCR-like antigen sensitivity outperforming TRuCs and CARs by efficiently exploiting the CD2/CD58 interaction}

The ability of the TCR to exploit adhesion interactions is known to depend on TCR signalling (46) and on structural features of the TCR/pMHC interaction, Including an intermembrane distance compatible with 
CD2/CD58 interactions $(21,47)$. Indeed, it has been shown that smaller CAR/antigen intermembrane distances produce higher $\mathrm{T}$ cell responses (48). Moreover, it has been suggested that signalling based on the $\mathrm{TCR} \zeta$-chain may limit CAR function. This has motivated the construction of CARs utilising the CD3 $\varepsilon$ chain $(16,17)$, TruCs that fuse the $\mathrm{scFv}$ directly to $\mathrm{CD} 3 \varepsilon$ for assembly with the complete TCR complex (18), and STARs that replace the variable domains of the $\alpha$ and $\beta$ TCR chains with those of the antibody (19) (Fig. 4A). However, a direct comparison of their antigen sensitivities has yet to be performed.

Using the D52N variable domains, we generated a set of chimeric receptors which increasingly resembled the endogenous TCR complex (Fig. 4A). We confirmed that they expressed at similar levels to the 1G4 TCR (Fig. S1). We next compared them to the TCR in terms of target cell killing and CD69 upregulation (Fig. 4B,C). We found that the STAR performed identically to the TCR whereas the TruC was intermediate between them and the standard $\zeta$-chain CAR, which itself performed similarly to the $\varepsilon$-chain CAR. To determine if adhesion interactions were involved in determining this antigen sensitivity hierarchy, we examined the impact of CD2 ligation. Similar to the antigen sensitivity hierarchy on cells, we found that the TCR and STAR were able to efficiently exploit CD2 with a $~ 100$-fold increase in sensitivity compared to an 25-fold increase by a TruC and the more modest 7-fold increase by CARs (Fig. 4D). Taken together, TCR-like chimeric receptors, and specifically STARs, are able to achieve antigen sensitivities comparable to the TCR and this capacity is predicted by their ability to exploit the CD2 adhesion interaction. 


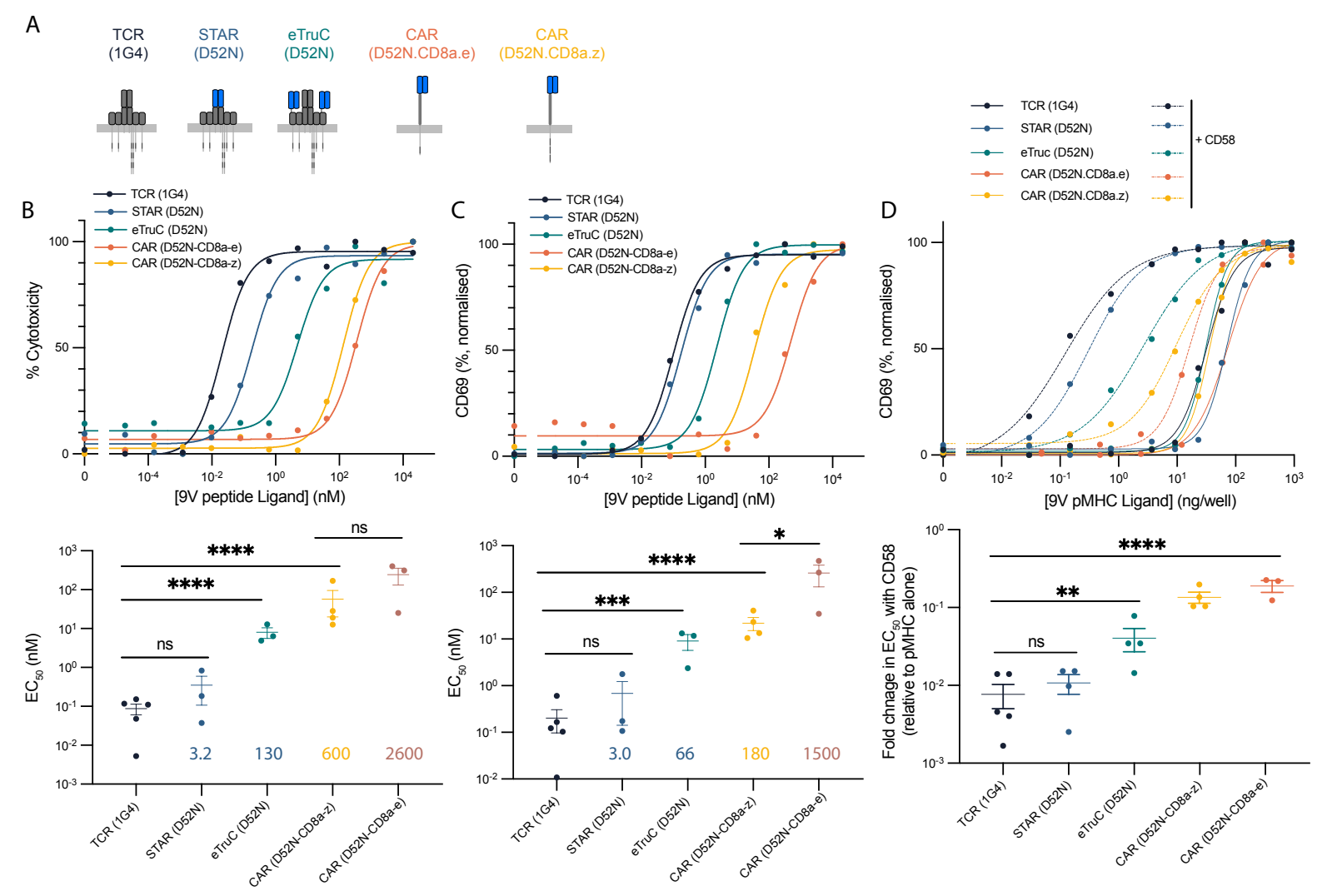

Figure 4: TCR-like chimeric antigen receptors are able to recapitulate the sensitivity of the TCR and exploit extrinsic adhesion interactions. (A) Schematic of 'TCR-like' engineered antigen receptors. (B) Representative dose-response curves showing LDH release (left) and CD69 (right) with corresponding $\mathrm{EC}_{50}$ values (bottom) when antigen is pulsed on T2 target cells. (C) Representative dose-response curves showing CD69 when antigen is presented as purified pMHC alone (solid lines) or with a fixed concentration of purified CD58 (dashed lines) with corresponding $\mathrm{EC}_{50}$ values. The $\mathrm{EC}_{50}$ values are compared using a one-way ANOVA (or two sample t-test) on log-transformed values. Abbreviations: $*=\mathrm{p}$-value $\leq 0.05$, $* *=$ p-value $\leq 0.01, * * *=\mathrm{p}$-value $\leq 0.001, * * * *=\mathrm{p}$-value $\leq 0.0001$.

\section{Discussion}

The antigen sensitivity of chimeric receptors that re-direct $\mathrm{T}$ cells to target abnormal (e.g. cancer) cells is critical for their efficacy, including control of potential escape mutants that express lower antigen levels $(1,2,6)$. Here, we have shown that all standard CAR formats, including $1^{\text {st }}$ and $2^{\text {nd }}$ generation CARs on common hinges (CD8a, CD28, and IgG1), have a >100-fold lower sensitivity to antigen relative to the TCR. This defect is not attributable to $\mathrm{T}$ cell exhaustion, antigen affinity, or co-receptor binding but rather to a failure of CARs to exploit co-stimulation by CD2 and LFA-1. Although fusing the CAR scFv directly to the $\mathrm{CD} 3 \varepsilon$ of the TCR complex (TruCs) improved antigen sensitivity, a complete grafting of the scFv variable domains in place of the variable domains of the TCR (STARs) was required to fully restore the antigen sensitivity observed with the TCR.

Although the antigen sensitivity of CARs is critically important, few studies have directly assessed it because it is difficult to systematically vary the antigen concentration on target cells. Consistent with 
our work, these studies have found a 100-1000-fold defect in CAR antigen sensitivity compared to the TCR. Harris et al (9) generated a CAR using the variable domains of a TCR showing that both $1^{\text {st }}$ and $2^{\text {nd }}$ generation CARs exhibited a $\sim 100$-fold lower antigen sensitivity. Wang et al (49) found similar defects when using primary $\mathrm{T}$ cells and, consistent with our findings, they report only modest impacts on antigen sensitivity for both the TCR and CARs when engaging the co-stimulation receptor CD28 (Fig. 2). Gudipati et al (10) report lower antigen sensitivities for CARs when using antigens on fluid-phase planar bilayers that contained a fixed concentration of ICAM-1, which is consistent with the ICAM-1 phenotype in our solid-phase stimulation system (Fig. 2). Therefore, our results are consistent with the published literature and localise the defect to a failure of CARs to exploit adhesion interactions.

The TCR employs a variety of mechanisms to achieve high antigen sensitivity, including the use of coreceptors $(\mathrm{CD} 4 / \mathrm{CD} 8)$ that bind $\mathrm{MHC}$ at a site that is independent of the TCR, and the adhesion receptors CD2 and LFA-1 (21). Co-receptors are not expected to contribute to CAR antigen sensitivity because CARs usually target non-MHC antigens and moreover, even when targeting pMHC, they bind with affinities that typically show co-receptor independent responses (50), which is the case for our D52N CAR targeting the NY-ESO-1 pMHC antigen. Although the adhesion receptor CD2 was initially shown to have a subtle role in murine $\mathrm{T}$ cells $(27,51)$, it is now appreciated to be important in human $\mathrm{T}$ cell activation $(30,52)$ including cancer cell killing (53) and viral evasion (54). In our solid-phase stimulation system we have found that CD2 engagement can increase antigen sensitivity by $>100$-fold for the TCR but $<10$-fold for CARs, and a similar pattern was observed for LFA-1 engagement albeit with lower fold-changes. These findings were also reproduced in the APC stimulation system but with lower fold-changes that may be associated with expression of other adhesion ligands on the U87 line (Fig. S5). Therefore, our data suggests that improving CAR antigen sensitivity will require optimisation of adhesion interactions and we have shown that one solution is to directly replace the TCR variable domains with those of the CAR. Consistent with this result, it has recently been shown that the resulting STARs outperform standard CARs in vivo. Although TruCs outperform CARs, we have found that they are less sensitive than the TCR and the STAR. Together, these findings suggest that efficiently exploiting extrinsic adhesion receptors may require both the full complement of TCR signalling and the endogenous structural features of the TCR.

In order to compare the antigen sensitivity of CARs with the TCR we have used T cells responding to MHC-presented peptide antigens. We used this system because the antigen density can be titrated to very low levels on APCs. In order to study antigen sensitivity using non-MHC antigens it is common to generate different APC populations that stably express different antigen levels. However, it is technically difficult to generate APCs with fewer than 100 antigens (55-57). While such a titration is possible using purified antigen, our results show that differences in antigen sensitivity may be underestimated unless purified ligands to CD2 and LFA-1 are also included in these systems. Indeed, this may explain the finding that different CARs targeting purified ROR1 in isolation had similar antigen sensitivities in a solid-phase stimulation $(<2$ fold) but large differences in their in vivo efficacy (11). While the use of MHC-presented peptide antigens is a powerful tool for studying antigen sensitivity, additional studies are needed to confirm the role of CD2 and LFA-1 in regulating sensitivity when targeting conventional antigens. In this regard, it is noteworthy that the ligand for murine CD2 is CD48, which has a much lower affinity and different expression pattern to the ligand for human CD2, which is CD58 (47). It follows that comparisons of antigen sensitivity in preclinical murine models may be misleading unless appropriately humanized (e.g. xenograft) mice are used. Consistent with our results, STARs have been shown to outperform CARs in tumour models, exhibiting a higher sensitivity to target antigens (57).

Although high antigen sensitivity is likely to be desired in many situations, there are clinical scenarios where the target antigen is over-expressed on abnormal cells and toxicity towards normal cells cannot be tol- 
erated $(58,59)$. In this situation, tuning the antigen sensitivity of CARs is desirable and this can be achieved by affinity-tuning the CAR $(45,59,60)$ or through transcriptional circuits $(61)$. Here, we have found that different CAR formats produce different antigen sensitivities. The hierarchy we report (STARS $>$ TruCs $>$ CAR (CD28 or CD8a hinge) > CAR (IgG1 hinge)) would suggest using standard CARs for targeting highly over-expressed antigens whereas STARs would be optimal for eliminating target cells independent of antigen levels. However, STARs would be more susceptible to immune escape by cancer cells that lose expression of accessory receptor ligands, such as CD58 and/or ICAM-1. A key advantage of tuning antigen sensitivity by changing the CAR format is that changes to the recognition domain (e.g. changing affinity) are not required, which avoids the potential of introducing mutations that impact the in vivo specificity of the recognition domain.

The TCR, and indeed the majority of CARs, belong to a large and diverse group of surface receptors known as immune receptors or Non-catalytic Tyrosine-phosphorylated Receptors (NTRs) (62). The mechanism by which these receptors convert extracellular ligand binding into intracellular phosphorylation and signaling, known as receptor triggering, remains debated. In the case of the TCR, allosteric activation has been proposed as a mechanism of triggering (63). Previous work has shown that grafting the variable domains from an antibody in place of the variable domains of the TCR produces a receptor that can activate $\mathrm{T}$ cells $(19,64)$ but it was unclear how this receptor compared to the native TCR. Here, we have shown that this chimeric receptor is indistinguishable from the TCR in terms of antigen sensitivity. This observation is difficult to reconcile with allosteric models of TCR activation given the very limited conservation between antibody and TCR variable domains. We note that conformational changes based on mechanical pulling forces would continue to operate with different variable domains.

In conclusion, chimeric receptors with antigen sensitivities that parallel the TCR can be engineered and this requires that they exploit accessory receptors, such as CD2 and LFA-1. This higher antigen sensitivity can allow $\mathrm{T}$ cells to continue to recognise cancer cells that downregulate the target antigen. However, cancer cells that downregulate both the target antigen and ligands to accessory receptors can escape T cell recognition suggesting that more complex circuits, including targeting multiple antigens, may be necessary (65). There is increasing interest in re-directing other immune cells using chimeric antigen receptors (66, 67). Our work suggests that engineering accessory receptor/ligand interactions may be necessary to achieve high sensitivity in other cell types that may not express the receptor CD2 for example. This may allow diverse cell types to achieve the same remarkable antigen sensitivity as the TCR.

\section{Acknowledgements}

We thank Marion H. Brown, Philipp Kruger, and John Nguyen for helpful discussion and reagents. We thank Linda Wooldridge and Christoph Renner for the DT227/8KA HLA-A2 and D52N CAR constructs, respectively.

\section{Funding}

The work was funded by a Wellcome Trust Senior Fellowship in Basic Biomedical Sciences (207537/Z/17/Z to OD), a Guy Newton Translational Grant (to OD), a Wellcome Trust PhD Studentship in Science (203737/Z/16/Z to JP), and the EPSRC \& BBSRC Doctoral Training Centre in Synthetic Biology (EP/L016494/1, supported J.B. and J.A.S.-F). 


\section{Materials \& Methods}

\section{T Cell Preparation}

Human CD8+ T cells were isolated from leukocyte cones purchased from the NHS Blood and Transplantation service. Isolation was performed using negative selection. Briefly: blood samples were incubated with Rosette-Sep Human CD8+ enrichment cocktail (Stemcell) at $150 \mu \mathrm{l} / \mathrm{ml}$ for 20 minutes. This was followed by a 3.1 fold dilution with PBS before layering on Ficoll Paque Plus (GE) at a 0.8:1.0 ficoll to sample ratio. Ficoll-Sample preparation was spun at $1200 \mathrm{xg}$ for 20 minutes at room temperature. Buffy coats were collected, washed and isolated cells counted. Cells were resuspended in complete RMPI (RPMI supplemented with $10 \%$ v/v FBS, 100 Units $/ \mathrm{ml}$ penicillin, $100 \mu \mathrm{g} / \mathrm{ml}$ streptomycin) with $50 \mathrm{U} / \mathrm{ml}$ of IL-2 (PeproTech) and CD3/CD28 Human T-activator Dynabeads (Thermo Fisher) at a 1:1 bead to cell ratio. At all times isolated human $\mathrm{CD} 8+\mathrm{T}$ cells were cultured at $37^{\circ} \mathrm{C}$ and $5 \% \mathrm{CO}_{2}$.

1 million cells in $1 \mathrm{ml}$ of media were subsequently transduced on the following day using lentivirus encoding for the various constructs (e.g., TCR), per the section on lentiviral transduction. On days 2 and 4 post-transduction, $1 \mathrm{ml}$ of media was exchanged and IL-2 was added to a final concentration of $50 \mathrm{U} / \mathrm{ml}$. Dynabeads were magnetically removed on day 5 post-transduction. T cells were further cultured at a density of 1 million cells $/ \mathrm{ml}$ and supplemented with $50 \mathrm{U} / \mathrm{ml} \mathrm{IL-2} \mathrm{every} \mathrm{other} \mathrm{day.} \mathrm{T} \mathrm{cells} \mathrm{were} \mathrm{used} \mathrm{between} 10$ and 16 days after transduction.

\section{Lentiviral Production}

HEK 293 T cells were seeded in DMEM supplemented with 10\% FBS and 1\% penicilin/streptomycin in 6-well plates to reach 60-80\% confluency on the following day. Cells were transfected with $0.25 \mu \mathrm{g}$ pRSV-

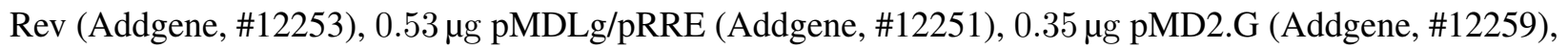
and $0.8 \mu \mathrm{g}$ of transfer plasmid using $5.8 \mu \mathrm{l}$ X-tremeGENE HP (Roche). Media was replaced after 16 hours and supernatant harvested after a further 24 hours by filtering through a $0.45 \mu \mathrm{m}$ cellulose acetate filter. Supernatant from one well of a 6-well plate was used to transduce 1 million T cells.

\section{Generating U87 knockout cell lines}

U87 cells (a kind gift of Vincenzo Cerundolo) were used to generate genetic knockouts for CD58, ICAM1, or both using CRISPR Cas9 RNP transfection. To generate CD58 KO cells, 50,000 U87 cells were seeded in a 24-well plate and transfected the next day using Lipofectamine CRISPRMAX Cas9 Transfection agent (Thermo Fisher), annealed crRNA:tracrRNA (TrueGuide CRISPR758411_CR, GTCAATGCACAAGTTAGTGT, Thermo Fisher; A35506 for tracrRNA, Thermo Fisher), and TrueCut Cas9 Protein v2 (Thermo Fisher, A36496) according to manufacturer's instructions. Cells were FAC sorted and this mixed population was used for all experiments. Sorted CD58 KO cells or WT U87 cells were used to generate CD58/ICAM1 double KO cells or ICAM1 KO cells, respectively using the same protocol as above. Specifically, cells were transfected with crRNA:tracrRNA (TrueGuide CRISPR845351_CR, GCTATTCAAACTGCCCTGAT, Thermo Fisher) and subsequently FAC sorted. Accutase (Biolegend 423201) was used to dissociate cells before screening or sorting with anti-CD58 (TS2/9, Invitrogen 12-0578-42) or anti-ICAM1 (HA58, Biolegend 
353114) to prevent potential digestion of CD58 or ICAM1 by trypsin. All cell lines showed similar expression of HLA-A2 by flow cytometry (clone BB7.2, Biolegend 343306).

\section{Co-culture with U87 Cells}

25000 U87 cells were seeded in a tissue culture treated flat-bottom 96 well plate and grown overnight. On the following day the media was removed from these cells and they were incubated with peptides prepared to the appropriate concentration in complete DMEM (DMEM supplemented with 10\% v/v FBS, $100 \mathrm{Units} / \mathrm{ml}$ penicillin, $100 \mu \mathrm{g} / \mathrm{ml}$ streptomycin) for 1 hour at $37^{\circ} \mathrm{C}$.

If blocking antibodies were used then the appropriate amount of $\mathrm{T}$ cells were incubated for 30 minutes prior to addition to the U87 cells with either anti-IgG1 $\chi$ Isotype control (BioLegend, Clone MOPC-21), anti-CD58 (BioLegend, Clone TS2/9) or anti-ICAM1 (eBioscience, Clone HA58) at a concentration of $10 \mu \mathrm{g} / \mathrm{ml}$. Alternatively, both anti-CD58 and anti-ICAM1 together at a concentration of $5 \mu \mathrm{g} / \mathrm{ml}$ each (total antibody concentration $10 \mu \mathrm{g} / \mathrm{ml})$.

Peptide containing media was then removed and 50,000 T cells per well were added. The co-culture was then spun for 2 minutes at $50 \mathrm{xg}$, and incubated for 4 hours at $37^{\circ} \mathrm{C}$. After this period a fraction of supernatant was removed for cytokine ELISAs and stored at $-20^{\circ} \mathrm{C}$. EDTA was added to the remaining supernatant (final concentration $2.5 \mu \mathrm{M}$ ) and cells were detached by pipetting.

Cells were stained in PBS 1\% BSA for CD45 (Clone HI30, dilution 1:200), CD69 (Clone FN50, dilution 1:200) and 4-1BB (Clone 4B4-1, dilution 1:200) as well as with PE-conjugated tetrameric pMHC (dilution 1:500). Stained cells were either analysed immediately or fixed with $1 \%$ formaldehyde in PBS and analysed on the following day.

T cells were discriminated from U87 cells by CD45 staining and/or an assessment of size and complexity. Single T cells were identified on the basis of size and subsequent analysis performed on this population.

\section{Co-culture with T2 Cells}

T2 cells were stained with $5 \mathrm{~mm}$ Tag-It Violet (BioLegend) following the manufacturer's protocol and then 60000 cells were seeded in a volume of $100 \mu \mathrm{L}$ per well in a V-bottom 96 well tissue culture plate. T2 cells were then incubated with $100 \mu \mathrm{L}$ of peptide dilution prepared to the desired concentration in complete RPMI for 1 hour at $37^{\circ} \mathrm{C}$. T2 cells were then washed, resuspended in $100 \mu \mathrm{l}$ of complete RPMI and transferred to a flat-bottom 96 well tissue culture plate.

Primary $\mathrm{T}$ cells were counted and re-suspended in fresh media such that there were 30000 receptor positive cells per $100 \mu \mathrm{l}$. This volume was then added to the T2 cells transferred previously.

As controls for the LDH assay additional wells were prepared in triplicate containing only $30000 \mathrm{~T}$ cells for each construct, or only $60000 \mathrm{~T} 2$ cells. Both with media to the same final volume as the co-cultured cells. Triplicate wells serving as volume correction and media controls were also prepared.

Plates were then spun at $50 \mathrm{x}$ for 2 minutes and incubated for 8 hours at $37^{\circ} \mathrm{C}$. After this period plates were spun again at $50 \mathrm{xg}$ for 2 minutes and a fraction of supernatant was removed for assessing LDH release. 
LDH release was assessed using CyQUANT LDH Cytotoxicity Assay Kits (Thermo Fisher) following the manufacturers protocol. EDTA was added to the remaining supernatant (final concentration $2.5 \mu \mathrm{M}$ ) and cells were detached by pipetting.

Cells were stained for CD69 (Clone FN50, dilution 1:200) as well as with pMHC tetramers (dilution 1:500). Stained cells were either analysed immediately or fixed with $1 \%$ formaldehyde in PBS and analysed on the following day.

$\mathrm{T}$ cells were discriminated from T2 cells by the absence of Tag-It Violet stain. Single T cells were identified on the basis of size and subsequent analysis performed on this population.

\section{Flow Cytometry}

Tetramers were produced using refolded monomeric biotinylated pMHC and streptavidin-PE (Biolegend) at a 1:4 molar ratio. Streptavidin-PE was added in 10 steps with a 10 minute incubation at room temperature between each addition. $0.05-0.1 \%$ sodium azide was added for preservation and tetramers were kept for up to 3 months at $4{ }^{\circ} \mathrm{C}$.

Samples were analysed using a BD LSR Fortessa X-20 (BD Biosciences) or CytoFLEX LX (Beckman Coulter) flow cytometer and data analysis was performed using FlowJo v10 (BD Biosciences).

\section{Electroporation}

868 TCR alpha and beta chains were amplified using PCR, adding a T7 promoter at the 5/ end. The resulting PCR product was 'cleaned up' using a NucleoSpin Gel and PCR clean-up kit (Macherey-Nagel). Capped and Poly(A) tailed mRNA was produced from this PCR product using a mMESSAGE mMACHINETM T7 ULTRA Transcription Kit (ThermoFisher). mRNA was collected by lithium chloride precipitation, quality checked by gel electrophoresis and stored in single use aliquots at $-80^{\circ} \mathrm{C}$.

For electroporation, $\mathrm{T}$ cells are collected and washed 3x with Opti-MEM (Gibco) and resuspended at a concentration of $25 \times 10^{6}$ cells $/ \mathrm{ml} .5 \times 10^{6}$ cells with $2 \mu \mathrm{g}$ per million cells of each of the RNA for the $\mathrm{TCR} \alpha, \beta$ and $\zeta$ chains. Cells were then aliquoted in $200 \mu \mathrm{l}$ into an electroporation cuvette (Cuvette Plus $2 \mathrm{~mm}$ gap BTX). Electroporation is performed using an ECM 830 Square Wave electroporation system (BTX) at $300 \mathrm{~V}$ for $2 \mathrm{~ms}$. Cells are then transferred to pre-warmed complete RPMI at a density of $1 \times 10^{6}$ cells $/ \mathrm{ml}$. Electroporated cells are used in assays 24 hours later.

\section{Plate Stimulation}

Pierce Streptavidin Coated High Capacity 96 well plates (Thermo Fisher) were washed with PBS and dilutions of biotinylated pMHC in PBS were added to each well in a $50 \mu$ l volume and incubated for 90 minutes at room temperature. Subsequently, plates were washed again with PBS and biotinylated accessory molecules (CD58, ICAM-1, CD86, CD70, 41BBL) were added at a fixed dose of $250 \mathrm{ng} /$ well in $50 \mu \mathrm{l}$. Plates were again incubated for 90 minutes and then washed with PBS. 
T cells were counted, washed in media and 75000 cells in $200 \mu \mathrm{l}$ were dispensed per well, Plates were spun for 2 minutes at $50 \mathrm{xg}$ and then incubated for 24 hours at $37^{\circ} \mathrm{C}$. Following this incubation a portion of supernatant was removed and stored for performing ELISAs. EDTA was added to the remaining supernatant (final concentration $2.5 \mathrm{mM}$ ) and cells were detached by pipetting. Collected cells were stained for CD45 (Clone HI30, dilution 1:200), CD69 (Clone FN50, dilution 1:200), 4-1BB (Clone 4B4-1, dilution 1:200) and with tetrameric PE-conjugated pMHC. Cells were analysed either immediately or 1 day later, following fixation with $1 \%$ formaldehyde in PBS.

\section{Immobilisation Assay}

Following a plate stimulation assay, after cells were collected, plates were washed 3 times with PBS 0.05\% TWEEN 20 ('PBST') and then stained with anti-HLA-A,B,C (clone W6/32, dilution 1:1000) in PBS for 2 hours at room temperature. Plates were then washed $3 \mathrm{x}$ with PBST and stained with secondary goat antimouse IgG IRDye 800CW (LI-COR) in PBS for a further 2 hours. Finally plates were washed one more time with PBST and then imaged using a LICOR Odyssey Sa (LI-COR). Integrated intensity per well is reported.

\section{Peptides}

Peptides were synthesised at a purity of $>95 \%$ (Peptide Protein Research, UK). 9V refers to a peptide derived from NY-ESO ${ }_{157-165}$ (SLLMWITQV), 4A is derived from the same sequence (SLLAWITQV), and SL9 refers to a peptide from HIV p17 GAG $77-85$ (SLYNTVATL).

\section{Protein Production}

HLA-A*02:01 heavy chain (UniProt residues 25-298) with a C-terminal BirA tag and $\beta_{2}$-microglobulin were expressed as inclusion bodies in E.coli, refolded in vitro as described in (68) together with the relevant peptide variants, and purified using size-exclusion chromatography on a Superdex S75 column (GE Healthcare, USA) in HBS-EP buffer (10 mM M HEPES pH 7.4, $150 \mathrm{mM} \mathrm{NaCl,} 3 \mathrm{mM}$ EDTA, 0.005\% v/v Tween-20). Purified pMHC was biotinylated using the BirA enzyme (Avidity, USA).

His-tagged, soluble extracellular domain (ECD) of human CD58 was produced either in Freestyle 293F suspension cells (Thermo Fisher) or adherent HEK 293T cells. His-tagged, soluble versions of the ECD of human ICAM1, 41BBL, CD70 and CD86 were produced using adherent HEK 293T cells. Freestyle 293F suspension cells were transfected using Freestyle MAX reagent, as previously reported (30). Adherent HEK 293T cells were transfected using Roche X-tremeGENE HP transfection reagent following the manufacturer's protocol. In both cases the resulting supernatant was filtered with a $0.45 \mu \mathrm{m}$ filter and proteins were then purified using Ni-NTA agarose columns. Biotinylation was either performed in vitro after purification, or in situ by co-transfection (final proportion 10\%) of a secreted BirA and adding $100 \mu \mathrm{M}$ Dbiotin to the growth media. Further purification and excess biotin removal was performed by size exclusion chromatography in HBS-EP.

D52N chains were produced as inclusion bodies in E. coli and refolded in vitro as described in (69), except that inclusion bodies were solubilised in $20 \mathrm{mM}$ Tris- $\mathrm{HCl}(\mathrm{pH} 8.0), 8 \mathrm{M}$ urea, $2 \mathrm{mM}$ DTT, refolding 
buffer contained $150 \mathrm{mM}$ Tris- $\mathrm{HCl}$ (pH 8.0), $3 \mathrm{M}$ urea, $200 \mathrm{mM}$ Arg-HCl, $0.5 \mathrm{mM}$ EDTA, $0.1 \mathrm{mM}$ PMSF, and the refolding mixture was dialysed against $10 \mathrm{mM}$ Tris- $\mathrm{HCl}(\mathrm{pH} 8.5)$. The $\mathrm{D} 52 \mathrm{~N}$ dimer was purified on anion-exchange chromatography on a HiTrap Q column, followed by size-exclusion chromatography on a Superdex S200 column (both from GE Healthcare).

D52N scFvs with the following sequence were produced by Absolute Antibody Ltd.

\section{D52N scFv:}

EVQLLESGGGLVQP GGSLRLSCAASGFTESTYQMSWVRQAP GKGLEW

VSGIVSSGGSTAYADSVKGRFTISRDNSKNTLYLQMNSLRAEDTAVY

YCAGELLPYYGMDVWGQGTTVTVSSAKTTPKLEEGEF SEARVQSELT

QPRSVSGSPGQSVTISCTGTERDVGGYNYVSWYQQHPGKAPKLIIHN

VIERSSGVPDRFSGSKSGNTASLTISGLQAEDEADYYCWSFAGGYYV

FGTGTDVTVLG

All purified proteins were aliquoted and stored at $-80^{\circ} \mathrm{C}$ until use.

\section{ELISAs}

Invitrogen Uncoated ELISA kits for TNF, IFN $\gamma$ and IL-2 (Thermo Fisher) were used following the manufacturer's protocol. Supernatants were either used immediately for ELISAs post-harvesting or stored at $-20^{\circ} \mathrm{C}$ for up-to 2 weeks. Supernatants were diluted using an empircally determined ratio before use in an ELISA so that quantities of assessed cytokines fell within the linear range of the kits.

\section{Surface Plasmon Resonance}

D52N-pMHC interactions were analysed on a Biacore T200 instrument (GE Healthcare Life Sciences) at $37^{\circ} \mathrm{C}$ and a flow rate of $30 \mu \mathrm{l} / \mathrm{min}$. Running buffer was HBS-EP (10 mM HEPES pH 7.4, $150 \mathrm{mM} \mathrm{NaCl}$, $3 \mathrm{mM}$ EDTA, $0.005 \% \mathrm{v} / \mathrm{v}$ Tween-20). Streptavidin was coupled to CM5 sensor chips using an amino coupling kit (GE Healthcare Life Sciences) to near saturation, typically 10,000-12,000 response units (RU). Biotinylated pMHCs $(47 \mathrm{kDa})$ were injected into the experimental flow cells (FCs) for different lengths of time to produce desired immobilisation levels (300-1000 RU). FC1 and FC3 were used as reference FCs for FC2 and FC4, respectively. Biotinylated ECD of CD58 (24 kDa $+25 \mathrm{kDa}$ glycosylation) was immobilised in the reference FCs at levels matching those of pMHCs. Excess streptavidin was blocked with two $40 \mathrm{~s}$ (D52N STAR) or $60 \mathrm{~s}$ (D52N scFv) injections of $250 \mu \mathrm{M}$ biotin (Avidity). Before injections of purified D52N, the chip surface was conditioned with eight injections of the running buffer. Dilution series of D52N were injected simultaneously in all FCs starting from the lowest concentration, which was injected again after the highest concentration to confirm stability of pMHC on the chip surface. The duration of injections ( 20 or $180 \mathrm{~s}$ ) was the same for conditioning and D52N injections. After every 2 or 3 D52N injections, buffer was injected to generate data for double referencing. In addition to subtracting the signal from the reference FC (single referencing), all D52N binding data were double referenced (70) versus the average of the closest buffer injections before and after D52N injection to correct for small differences in signal between flow cells. D52N binding versus D52N concentration was fitted with the following model: $B=B_{\max } \cdot \frac{[D 52 N]}{K_{D}+[D 52 N]}$, where $B$ is the response (binding) and $B_{\max }$ is the maximal binding. 


\section{Analysis}

Unless otherwise stated all statistics are performed on log-transformed data. Statistics were computed with GraphPad Prism 8 (GraphPad Software) or Excel (Microsoft). Curve fitting was performed using the robust nonlinear regression functionality in GraphPad Prism or MATLAB (MathWorks) and the $E C_{50}$ extracted from the fitted curves. Data was excluded from analysis if the computed fit was reported as 'ambiguous' in Prism, if the fit did not converge in 1000 iterations, or if the computed $E C_{50}$ would be outside of the tested ligand range. 


\section{References}

1. June CH, O'Connor RS, Kawalekar OU, Ghassemi S, Milone MC (2018) CAR T cell immunotherapy for human cancer. Science 359:1361-1365.

2. Exley AR, McBlane J (2021) Regulating innovation in the early development of cell therapies. Immunotherapy Advances 1:1-18.

3. Fry TJ, et al. (2018) CD22-targeted CAR T cells induce remission in B-ALL that is naive or resistant to CD19-targeted CAR immunotherapy. Nature Medicine 24:20-28.

4. Park JH, et al. (2018) Long-Term Follow-up of CD19 CAR Therapy in Acute Lymphoblastic Leukemia. New England Journal of Medicine 378:449-459.

5. Shah NN, Fry TJ (2019) Mechanisms of resistance to CAR T cell therapy. Nature Reviews Clinical Oncology 16:372-385.

6. Majzner RG, Mackall CL (2018) Tumor antigen escape from car t-cell therapy. Cancer Discovery 8:1219-26.

7. Brudno JN, et al. (2018) T cells genetically modified to express an anti-B-Cell maturation antigen chimeric antigen receptor cause remissions of poor-prognosis relapsed multiple myeloma. Journal of Clinical Oncology 36:2267-2280.

8. Cohen A, et al. (2019) B cell maturation antigen - specific CAR T cells are clinically active in multiple myeloma The Journal of Clinical Investigation B cell maturation antigen - specific CAR T cells are clinically active in multiple myeloma. The Journal of Clinical Investigation 129:2210-2221.

9. Harris DT, et al. (2018) Comparison of t cell activities mediated by human tcrs and cars that use the same recognition domains. The Journal of Immunology 200:1088-1100.

10. Gudipati V, et al. (2020) Inefficient car-proximal signaling blunts antigen sensitivity. Nature Immunology 21:848-856.

11. Salter AI, et al. (2021) Comparative analysis of TCR and CAR signaling informs CAR designs with superior antigen sensitivity and in vivo function. Science Signaling 14.

12. Eshhar Z, Waks T, Gross G, Schindler DG (1993) Specific activation and targeting of cytotoxic lymphocytes through chimeric single chains consisting of antibody-binding domains and the $\gamma$ or $\zeta$ subunits of the immunoglobulin and T-cell receptors. Proceedings of the National Academy of Sciences of the United States of America 90:720-724.

13. Maher J, Brentjens RJ, Gunset G, Rivière I, Sadelain M (2002) Human T-lymphocyte cytotoxicity and proliferation directed by a single chimeric TCR $\zeta / C D 28$ receptor. Nature Biotechnology 20:70-75.

14. Imai C, et al. (2004) Chimeric receptors with 4-1BB signaling capacity provoke potent cytotoxicity against acute lymphoblastic leukemia. Leukemia 18:676-684.

15. Khalil DN, Smith EL, Brentjens RJ, Wolchok JD (2016) The future of cancer treatment: immunomodulation, CARs and combination immunotherapy. Nature Reviews Clinical Oncology.

16. Hartl FA, et al. (2020) Noncanonical binding of Lck to CD3 $\epsilon$ promotes TCR signaling and CAR function. Nature Immunology 21:902-913. 
17. $\mathrm{Wu} \mathrm{W}$, et al. (2020) Multiple signaling roles of $\operatorname{cd} 3 \varepsilon$ and its application in car-t cell therapy. Cell $182: 855-871$.

18. Baeuerle PA, et al. (2019) Synthetic truc receptors engaging the complete t cell receptor for potent anti-tumor response. Nature communications 10:1-12.

19. Liu Y, et al. (2021) Chimeric star receptors using tcr machinery mediate robust responses against solid tumors. Science Translational Medicine 13.

20. Huang J, et al. (2013) A Single Peptide-Major Histocompatibility Complex Ligand Triggers Digital Cytokine Secretion in CD4+ T Cells. Immunity pp 1-12.

21. Siller-Farfán JA, Dushek O (2018) Molecular mechanisms of T cell sensitivity to antigen. Immunological Reviews 285:194-205.

22. Holst J, et al. (2008) Scalable signaling mediated by $\mathrm{T}$ cell antigen receptor-CD3 ITAMs ensures effective negative selection and prevents autoimmunity. Nature immunology 9:658-66.

23. James JR (2018) Tuning ITAM multiplicity on T cell receptors can control potency and selectivity to ligand density. Science Signaling 11.

24. Purbhoo MA, et al. (2001) The Human CD8 Coreceptor Effects Cytotoxic T Cell Activation and Antigen Sensitivity Primarily by Mediating Complete Phosphorylation of the T Cell Receptor Chain. Journal of Biological Chemistry 276:32786-32792.

25. Irvine DJ, Purbhoo MA, Krogsgaard M, Davis MM (2002) Direct observation of ligand recognition by T cells. Nature 419:845-849.

26. Bachmann MF, et al. (1997) Distinct Roles for LFA-1 and CD28 during Activation of Naive T Cells : Adhesion versus Costimulation. Immunity 7:549-557.

27. Bachmann MF, Barner M, Kopf M (1999) CD2 sets quantitative thresholds in T cell activation. Journal of Experimental Medicine 190:1383-1392.

28. Cheadle EJ, et al. (2012) Ligation of the CD2 co-stimulatory receptor enhances IL-2 production from first-generation chimeric antigen receptor T cells. Gene Therapy 19:1114-1120.

29. Aleksic M, et al. (2010) Dependence of T cell antigen recognition on T cell receptor-peptide MHC confinement time. Immunity 32:163-74.

30. Pettmann J, et al. (2021) The discriminatory power of the t cell receptor. eLife 10:1-42.

31. Maus MV, et al. (2016) An MHC-restricted antibody-based chimeric antigen receptor requires TCR-like affinity to maintain antigen specificity. Molecular Therapy - Oncolytics 3:16023.

32. Rapoport AP, et al. (2015) Ny-eso-1-specific tcr-engineered t cells mediate sustained antigen-specific antitumor effects in myeloma. Nature medicine 21:914-921.

33. Chmielewski M, Hombach A, Abken H (2011) Cd28 cosignalling does not affect the activation threshold in a chimeric antigen receptor-redirected t-cell attack. Gene therapy 18:62-72.

34. Dushek O, et al. (2011) Antigen potency and maximal efficacy reveal a mechanism of efficient T cell activation. Science Signaling 4:ra39. 
35. Lever M, et al. (2016) A minimal signalling architecture explains the $\mathrm{T}$ cell response to a 1,000,000-fold variation in antigen affinity and dose. Proc Natl Acad Sci USA pp E6630-E6638.

36. Abu-Shah E, et al. (2020) Human CD8 + T Cells Exhibit a Shared Antigen Threshold for Different Effector Responses. The Journal of Immunology 205:1503-1512.

37. Trendel N, et al. (2021) Perfect adaptation of CD8+ T cell responses to constant antigen input over a wide range of affinity is overcome by costimulation.

38. Nguyen J, Pettmann J, Kruger P, Dushek O (2021) Differential impact of TNFRSF co-stimulation on CD8+ T cell cytokine production by feedback control of surface receptor expression. bioRxiv.

39. Pettmann J, et al. (2020) T cells exhibit unexpectedly low discriminatory power and can respond to ultra-low affinity peptide-mhe ligands. bioRxiv.

40. Long AH, et al. (2015) 4-1BB costimulation ameliorates $\mathrm{T}$ cell exhaustion induced by tonic signaling of chimeric antigen receptors. Nature Medicine 21:581-90.

41. Oh J, et al. (2019) Single variable domains from the T cell receptor $\beta$ chain function as mono- and bifunctional CARs and TCRs. Scientific Reports 9:1-12.

42. Lynn RC, et al. (2019) c-Jun overexpression in CAR T cells induces exhaustion resistance. Nature 576:293-300.

43. Thomas S, et al. (2011) Human T cells expressing affinity-matured TCR display accelerated responses but fail to recognize low density of MHC-peptide antigen. Blood 118:319-29.

44. Irving M, et al. (2012) Interplay between $\mathrm{T}$ cell receptor binding kinetics and the level of cognate peptide presented by major histocompatibility complexes governs CD8+ $\mathrm{T}$ cell responsiveness. The Journal of biological chemistry 287:23068-78.

45. Drent E, et al. (2017) A Rational Strategy for Reducing On-Target Off-Tumor Effects of CD38-Chimeric Antigen Receptors by Affinity Optimization. Molecular Therapy 25:1946-1958.

46. Brownlie RJ, Zamoyska R (2013) T cell receptor signalling networks: branched, diversified and bounded. Nature Reviews Immunology 13:257-269.

47. Davis SJ, Ikemizu S, Wild MK, van der Merwe Pa (1998) CD2 and the nature of protein interactions mediating cell-cell recognition. Immunological reviews 163:217-36.

48. Li J, et al. (2017) Membrane-Proximal Epitope Facilitates Efficient T Cell Synapse Formation by AntiFcRH5/CD3 and Is a Requirement for Myeloma Cell Killing. Cancer Cell 31:383-395.

49. Wang X, et al. (2021) Extensive functional comparisons between chimeric antigen receptors and T cell receptors highlight fundamental similarities. Molecular Immunology 138:137-149.

50. Holler PD, Kranz DM (2003) Quantitative analysis of the contribution of TCR/pepMHC affinity and CD8 to T cell activation. Immunity 18:255-64.

51. van der Merwe Pa (1999) A subtle role for CD2 in T cell antigen recognition. The Journal of Experimental Medicine 190:1371-1374.

52. Leitner J, Herndler-Brandstetter D, Zlabinger GJ, Grubeck-Loebenstein B, Steinberger P (2015) CD58/CD2 Is the Primary Costimulatory Pathway in Human CD28 CD8 + T Cells . The Journal of Immunology 195:477-487. 
53. Patel SJ, et al. (2017) Identification of essential genes for cancer immunotherapy. Nature 548:537-542.

54. Wang ECY, et al. (2018) Suppression of costimulation by human cytomegalovirus promotes evasion of cellular immune defenses. Proceedings of the National Academy of Sciences 115:4998-5003.

55. Walker AJ, et al. (2017) Tumor Antigen and Receptor Densities Regulate Efficacy of a Chimeric Antigen Receptor Targeting Anaplastic Lymphoma Kinase. Molecular Therapy 25:2189-2201.

56. Salzer B, et al. (2020) Engineering AvidCARs for combinatorial antigen recognition and reversible control of CAR function. Nature Communications 11:1-16.

57. Liu Y, et al. (2021) Chimeric STAR Receptors Utilizing TCR Machinery Mediate Robust Response to Solid Tumors. Science Translational Medicine eabb5191.

58. Morgan Ra, et al. (2010) Case report of a serious adverse event following the administration of $\mathrm{T}$ cells transduced with a chimeric antigen receptor recognizing ERBB2. Molecular therapy 18:843-851.

59. Liu X, et al. (2015) Affinity-tuned ErbB2 or EGFR chimeric antigen receptor T cells exhibit an increased therapeutic index against tumors in mice. Cancer Research 75:3596-3607.

60. Caruso HG, et al. (2015) Tuning sensitivity of CAR to EGFR density limits recognition of normal tissue while maintaining potent antitumor activity. Cancer Research 75:3505-3518.

61. Hernandez-Lopez RA, et al. (2021) T cell circuits that sense antigen density with an ultrasensitive threshold. Science 371:1166-1171.

62. Dushek O, Goyette J, van der Merwe PA (2012) Non-catalytic tyrosine-phosphorylated receptors. Immunological Reviews 250:258-276.

63. van der Merwe PA, Dushek O (2011) Mechanisms for T cell receptor triggering. Nature Reviews Immunology 11:47-55.

64. Kuwana Y, et al. (1987) Expression of chimeric receptor composed of immunoglobulin-derived V resions and T-cell receptor-derived $\mathrm{C}$ regions. Biochemical and Biophysical Research Communications 149:960-968.

65. Rafiq S, Hackett CS, Brentjens RJ (2020) Engineering strategies to overcome the current roadblocks in CAR T cell therapy. Nature Reviews Clinical Oncology 17:147-167.

66. Morrissey MA, et al. (2018) Chimeric antigen receptors that trigger phagocytosis. eLife 7:1-21.

67. Klichinsky M, et al. (2020) Human chimeric antigen receptor macrophages for cancer immunotherapy. Nature Biotechnology 38:947-953.

68. Achour A, et al. (1999) Murine class i major histocompatibility complex h-2dd: expression, refolding and crystallization. Acta Crystallographica Section D: Biological Crystallography 55:260-262.

69. Boulter JM, et al. (2003) Stable, soluble t-cell receptor molecules for crystallization and therapeutics. Protein engineering 16:707-711.

70. Myszka DG (1999) Improving biosensor analysis. Journal of Molecular Recognition 12:279-284.

71. Uhlén M, et al. (2015) Tissue-based map of the human proteome. Science 347. 


\title{
Supplementary Information: \\ Inefficient exploitation of accessory receptors reduces the sensitivity of chimeric antigen receptors
}

\author{
Jake Burton $^{1}$, Jesús A. Siller-Farfán ${ }^{1 \uparrow}$, Johannes Pettmann ${ }^{1}$, Benjamin Salzer ${ }^{1 \dagger}$, \\ Mikhail Kutuzov ${ }^{1}$, P. Anton van der Merwe ${ }^{1}$, Omer Dushek ${ }^{1 \uparrow}$ \\ ${ }^{1}$ Sir William Dunn School of Pathology, University of Oxford, OX1 3RE, Oxford, UK \\ †Present address: St. Anna Children's Cancer Research Institute (CCRI), 1090, Vienna, Austria \\ \Corresponding authors
}

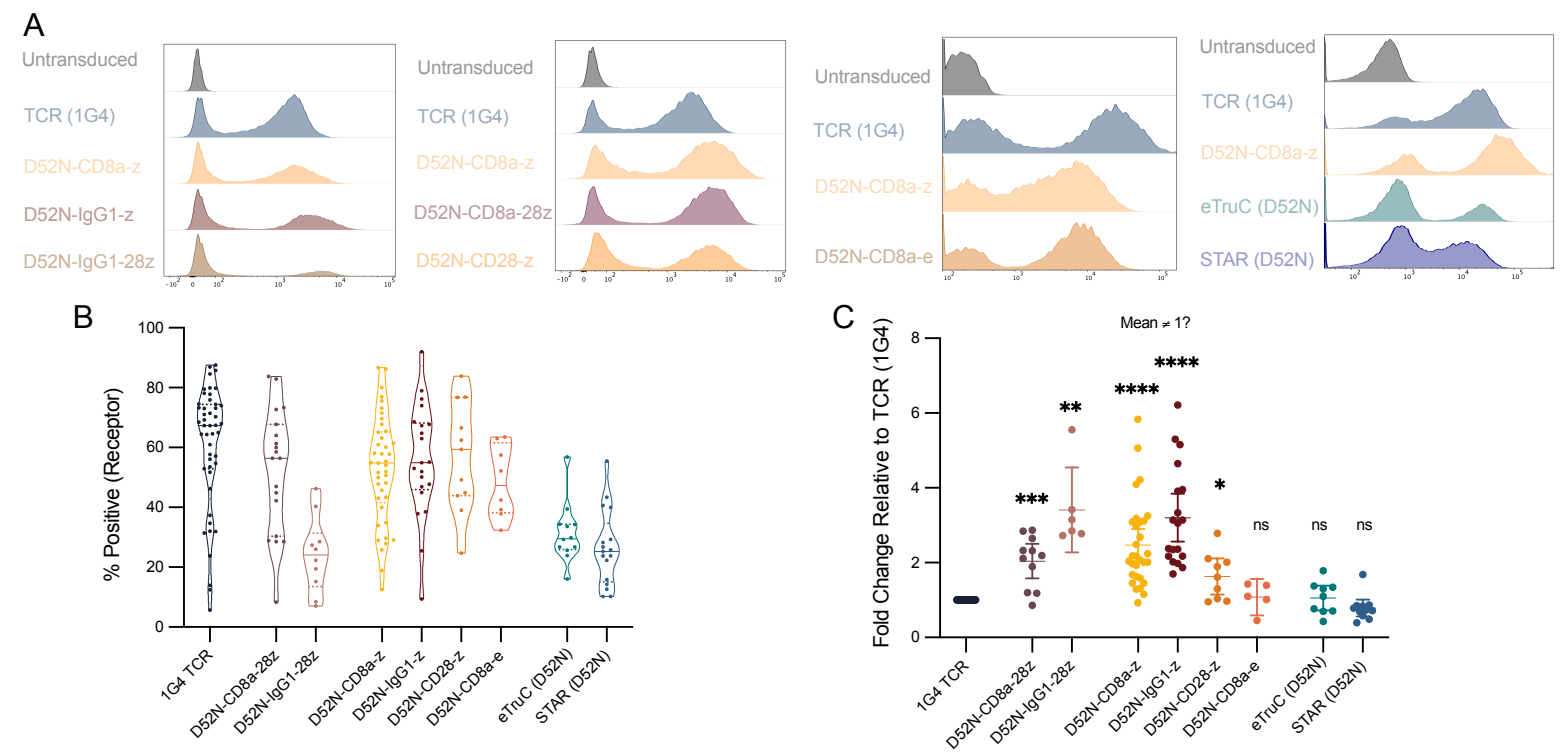

Figure S1: Surface expression of chimeric receptors was similar or higher than the TCR. (A) Representative flow cytometry histograms showing surface expression of the indicated surface receptor using fluorescent tetramers of the $9 \mathrm{~V} \mathrm{pMHC}$. Each column is a different experiment that included the TCR and the indicated chimeric receptors. (B) The percent of T cells expressing the indicated receptor (transduction efficiency). (C) The fold-change in the gMFI (expression level) relative to the TCR for the indicated receptors. A one-sample t-test is used to compare each chimeric receptor to the expression of the TCR (1.0) on log-transformed values. Abbreviations: $*=\mathrm{p}$-value $\leq 0.05, * *=\mathrm{p}$-value $\leq 0.01, * * *=\mathrm{p}$-value $\leq 0.001, * * * *$ $=\mathrm{p}$-value $\leq 0.0001$. 
A

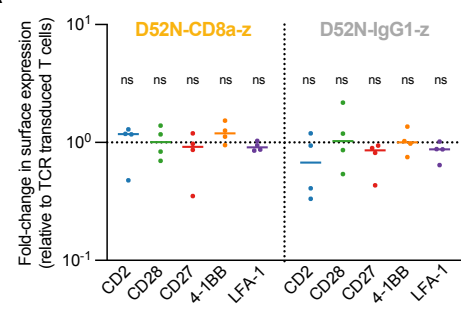

B
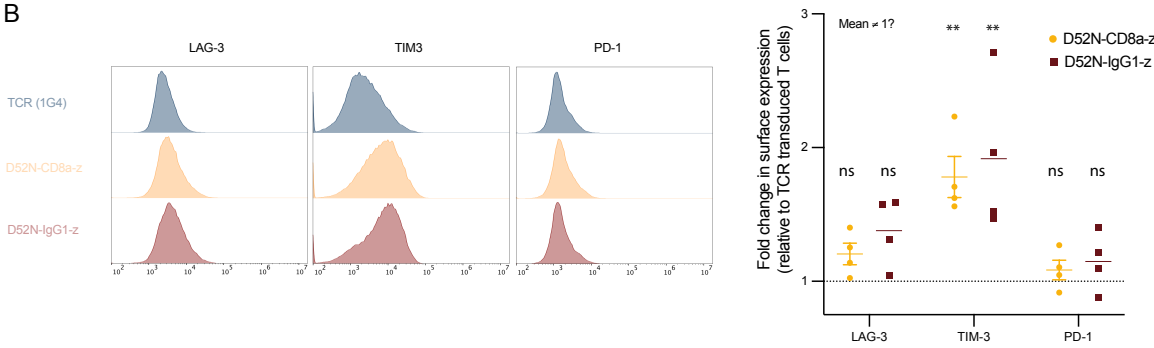

C
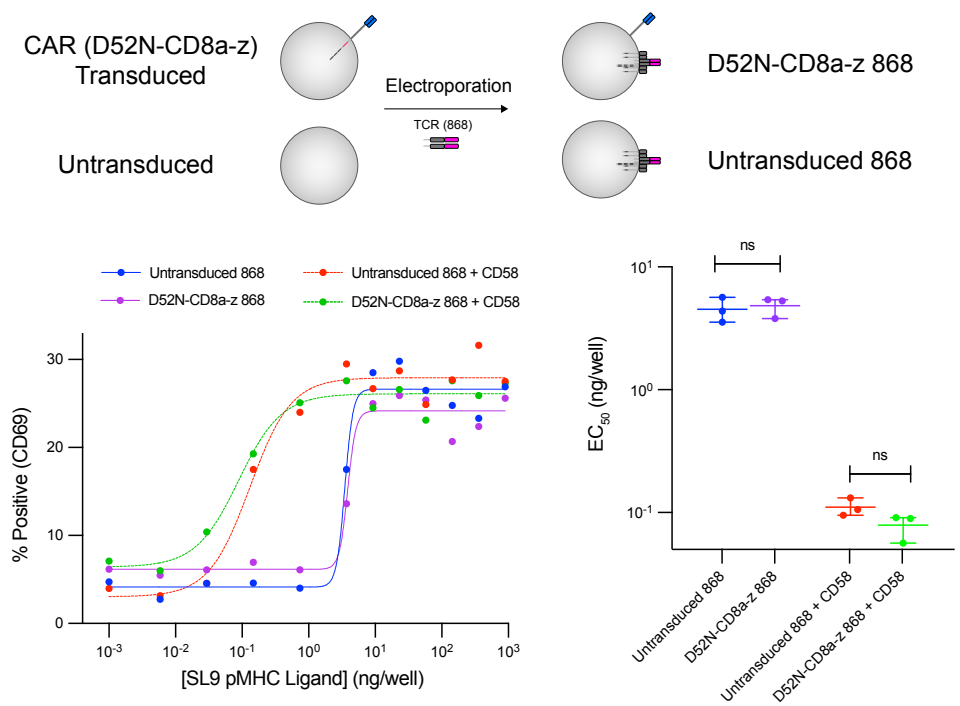

Figure S2: The CAR antigen sensitivity defect is not a result of exhaustion induced by tonic signalling. (A) Surface expression of the indicated co-stimulation receptors on T cells transduced with the TCR relative to $\mathrm{T}$ cells transduced with the indicated CARs. (B) Surface expression of the indicated co-inhibitory receptors on $\mathrm{T}$ cells transduced with the TCR relative to $\mathrm{T}$ cells transduced with the indicated CARs. (C) CAR transduced or untransduced T cells are electroporated with the 868 TCR specific to the SL9 peptide antigen before being stimulated by purified SL9 pMHC with or without CD58. A one-sample t-test is used to obtain a p-value for the null hypothesis that the indicated surface receptor expression differs from 1.0 on log-transformed values (panel $\mathrm{A}$ and panel $\mathrm{B}$, right) and a two-sample t-test is used to compare logtransformed $E C_{50}$ (panel $\mathrm{C}$, bottom right). Abbreviations: $*=\mathrm{p}$-value $\leq 0.05, * *=\mathrm{p}$-value $\leq 0.01, * * *=$ $\mathrm{p}$-value $\leq 0.001, * * * *=\mathrm{p}$-value $\leq 0.0001$. 
A Recombinant D52N scFv injected over 9V pMHC

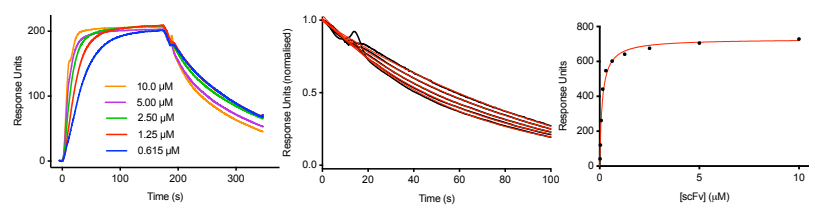

Matched Affinity $\left(\mathrm{K}_{\mathrm{D}} \sim 7 \mu \mathrm{M}\right)$

Recombinant D52N scFv injected over 4A pMHC
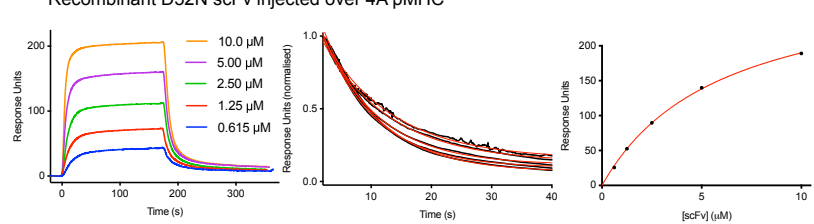

D52N scFV

$1 \mathrm{G} 4 \mathrm{TCR}$

\begin{tabular}{|c|c|c|c|c|c|}
\hline pMHC & $\mathrm{k}_{\text {on }}\left(\mu \mathrm{M}^{-1} \mathrm{~s}^{-1}\right)$ & $\mathrm{k}_{\text {off }}\left(\mathrm{s}^{-1}\right)$ & $K_{D}(\mu M)$ & $\mathrm{n}$ & $\mathrm{K}_{\mathrm{D}}(\mu \mathrm{M})$ \\
\hline 9V (SLLMWITQV) & 0.087 & $0.013 \pm 0.001$ & $0.15 \pm 0.03$ & 3 & $7.2 \pm 0.5$ \\
\hline 4A (SLLAWITQV) & 0.021 & $0.15 \pm 0.07$ & $7.0 \pm 1.4$ & 2 & $299 \pm 49$ \\
\hline
\end{tabular}

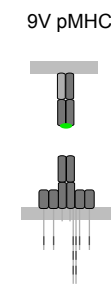

$1 \mathrm{G} 4$ TCR
4A pMHC

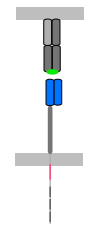

D52N CAR
B

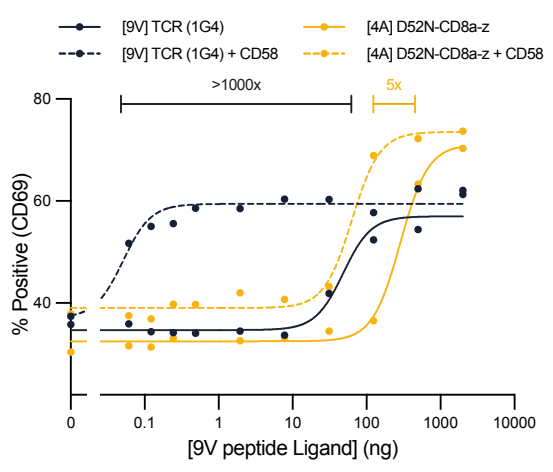

D

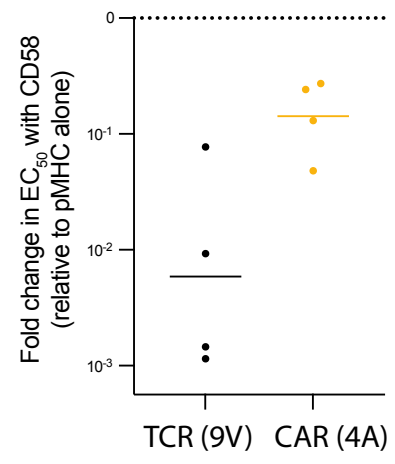

C

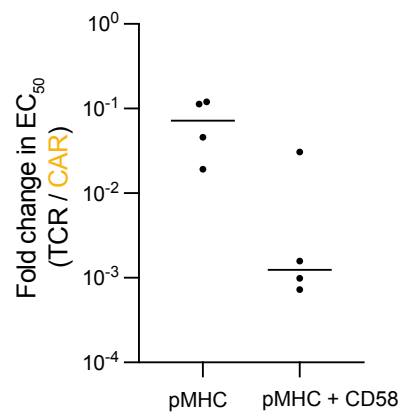

$E$

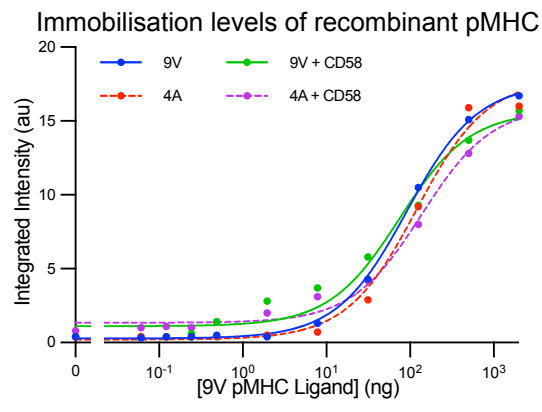

Figure S3: Matching the antigen affinity of the TCR and CAR increases the antigen sensitivity defect of CARs. (A) Binding between purified D52N scFv and the 9V (top) or 4A (bottom) pMHCs measured by surface plasmon resonance (SPR) showing the full sensogram (left), dissociation phase (middle) and steady state binding response (right). The kinetic $k_{\text {off }}$ and equilibrium $\mathrm{K}_{\mathrm{D}}$ are obtained by fitting the dissociation phase and steady-state binding response, respectively (red line is model fit). The kinetic $k_{\text {on }}$ is derived from $\mathrm{K}_{\mathrm{D}}$ and $k_{\text {off. }}$. The $\mathrm{K}_{\mathrm{D}}$ values for the TCR are obtained from previous work (30). (B) Representative dose-response for the TCR recognising the $9 \mathrm{~V}$ pMHC and for the CAR recognising the 4A pMHC with (dashed line) or without (solid line) purified CD58 (250 ng/well). C Fold-change in EC $_{50}$ between the TCR recognising $9 \mathrm{~V}$ and the $\mathrm{CAR}$ recognising $4 \mathrm{~A}$. D Fold-change in $\mathrm{EC}_{50}$ induced by the addition of CD58 for the indicated pMHC and antigen receptor. (E) Levels of presented pMHC for each condition as detected by the conformationally sensitive W6/32 antibody. 
A

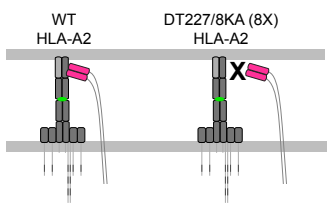

B

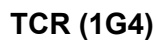

D
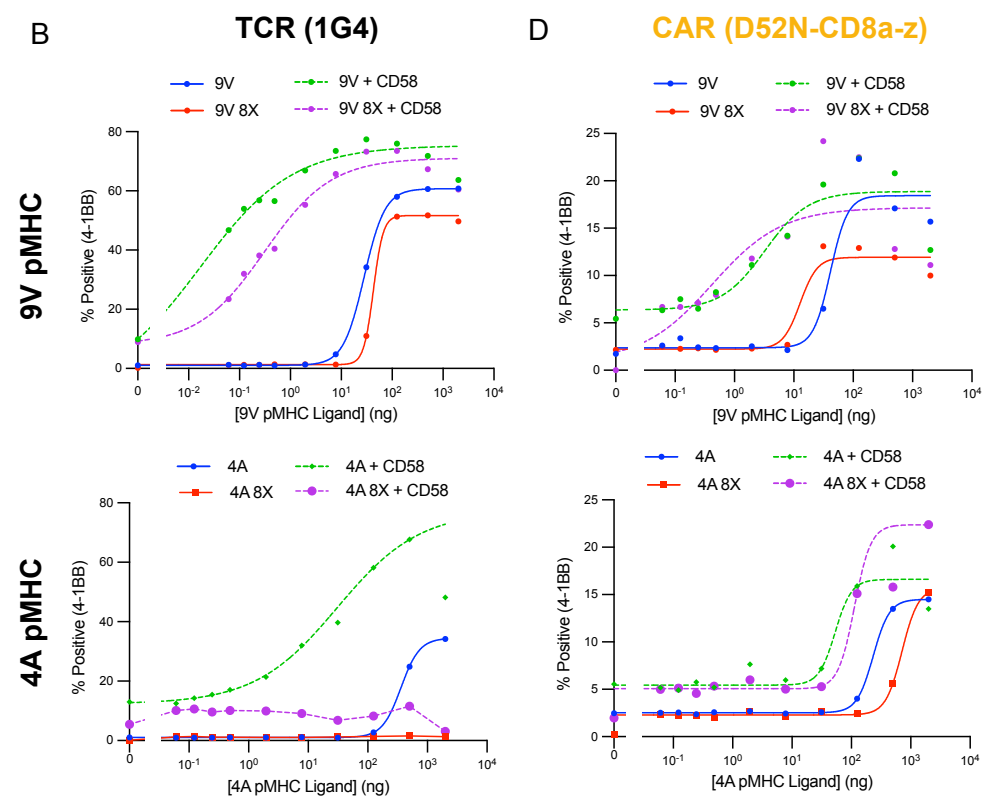

C

TCR (1G4)

E

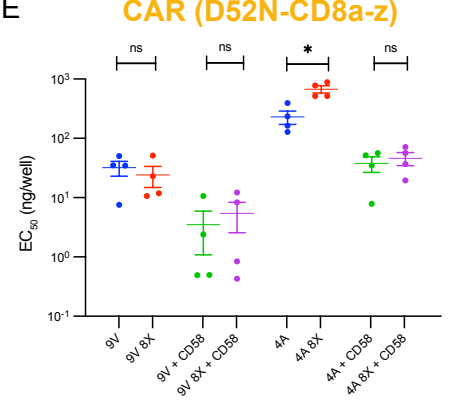

Figure S4: The CAR antigen sensitivity defect is independent of the CD8 co-receptor. (A) The DT227/8KA mutations in the HLA-A2 heavy-chain prevent binding by the CD8 co-receptor (referred to as 8X). (B-E) Representative dose-response curves (B,D) and summary measures (C,E) for the $9 \mathrm{~V}$ and $4 \mathrm{~A}$ pMHC variants with or without CD58 for the TCR $(B, C)$ and the CAR $(D, E)$. A t-test is used to compare the $E C_{50}$ values on log-transformed data. Abbreviations: $*=\mathrm{p}$-value $\leq 0.05, * *=\mathrm{p}$-value $\leq 0.01, * * *=$ $\mathrm{p}$-value $\leq 0.001, * * * *=\mathrm{p}$-value $\leq 0.0001$. 
A
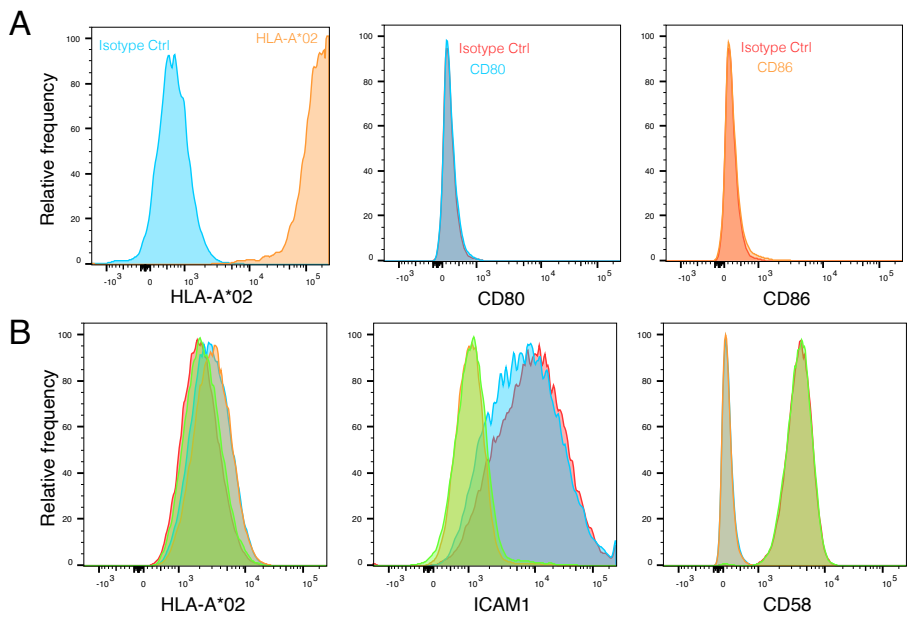

$\mathrm{C}$

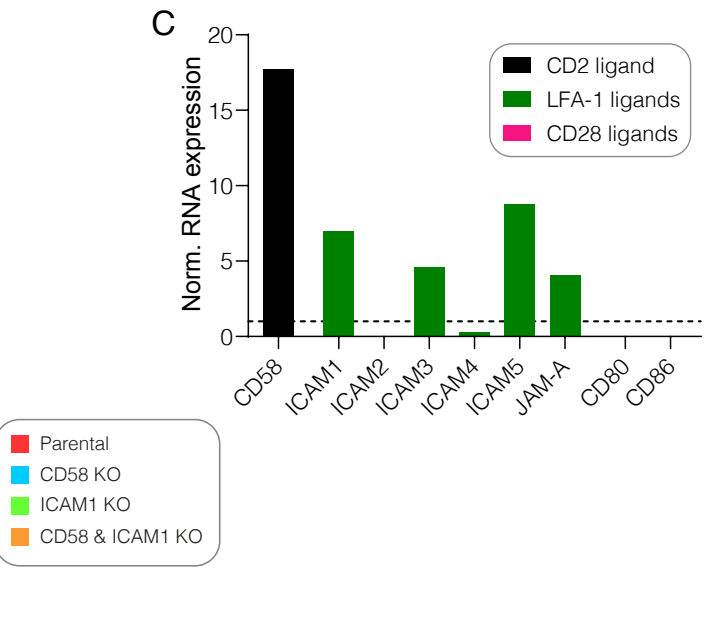

Figure S5: Expression of accessory receptor ligands on the U87 glioblastoma cell line. (A) Expression of HLA-A*02 (left), CD80 (middle), and CD86 (right) on parental U87 cells. (B) Expression of HLA-A*02 (left), ICAM-1 (middle), and CD58 (right) on the parental U87 cells or the indicated knockout cell lines. (C) Expression of the indicated molecule by RNA as reported in the Human Cell Atlas (71). 


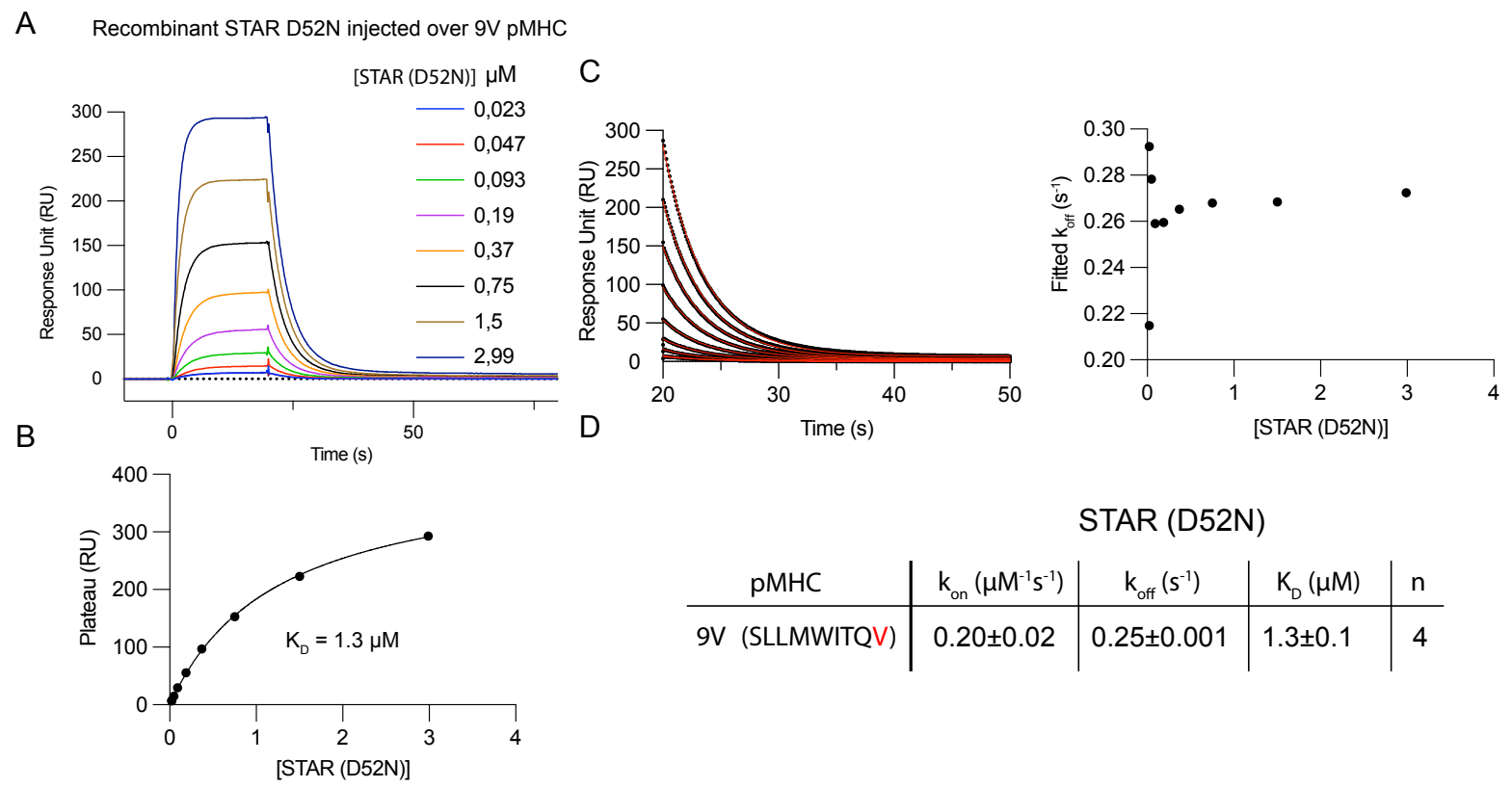

Figure S6: The binding affinity and kinetics between recombinant STAR (D52N) and 9V pMHC. (A) Representative binding between purified D52N STAR at the indicated concentration injected over a surface with immbolised 9V pMHC measured by surface plasmon resonance (SPR) showing the full sensogram. (B) The equilibrium $\mathrm{K}_{\mathrm{D}}$ is obtained by fitting the steady-state response. (C) The kinetic $k_{\text {off }}$ for each experiment is obtained by fitting the dissociation phase (left) and averaging the values for different concentrations (right). (D) Summary of binding constants with the kinetic $k_{\text {on }}$ determined for each experiment using $\mathrm{K}_{\mathrm{D}}$ and $k_{\text {off. }}$ 